\title{
Divergent synthesis of (+)-tanikolide and its analogues employing stereoselective rhodium(II)-catalyzed reaction
}

\author{
Hikari Jinnouchi, Hisanori Nambu, Tomoya Fujiwara, Takayuki Yakura* \\ Graduate School of Medicine and Pharmaceutical Sciences, University of Toyama, Sugitani, Toyama 930-0194, Japan
}

\section{ARTICLE INFO}

\section{Article history:}

Received

Received in revised form

Accepted

Available online

\section{Keywords:}

Divergent synthesis

Oxonium ylide

Rearrangement

Rhodium

Lactonization

\section{ABSTRACT}

In this study, we described the divergent synthesis of $(+)$-tanikolide and its analogues, such as $(4 S)$ - and (4R)-hydroxytanikolides, and nortanikolide, employing a stereoselective dirhodium(II)-catalyzed reaction to construct the quaternary chiral center of tanokolides. The key steps involve (a) a dirhodium(II)-catalyzed oxonium ylide formation-[2,3]-sigmatropic rearrangement, (b) an $\mathrm{N}$-heterocyclic carbene-catalyzed ring-expansion lactonization of tetrahydrofurfural, or (c) an oxidative cleavage of tetrahydrofuran-5-methanol to $\gamma$-lactone using a 2-iodobenzamide catalyst. This route would provide high flexibility for analogue synthesis because the long side chain can be introduced at a later stage in the synthesis.

2017 Elsevier Ltd. All rights reserved.

\section{Introduction}

$(+)$-Tanikolide (1, Fig. 1) and (-)-malyngolide, which are $\delta$ lactones with alkyl long chains and hydroxymethyl groups at $\mathrm{C} 5$, were isolated from the marine cyanobacterium Lyngbya majuscula collected from Tanikeli Island, Madagascar ${ }^{1}$ and the shallow water variety of Lyngbya Majuscula, ${ }^{2}$ respectively. Interestingly, the stereochemistries of these natural products are opposite in comparison with C5 and alkyl side chains of different lengths. Additionally, malyngolide possesses a methyl group at C2. With respect to their biological activities, tanikolide exhibits strong toxicity against brine shrimp and snails and malyngolide displays an antimicrobial activity against Mycobacterium smegmatis and Streptococcus pyogenes. ${ }^{2}$ Furthermore, tanikolide exhibits antifungal activity against Candida albicans, ${ }^{1}$ while malyngolide shows no activity against it. ${ }^{1}$ Since there is a clear difference in their biological activities in spite of their structural similarities, their analogues, possessing slightly different substituents, may lead to new promising candidates for drug discovery.

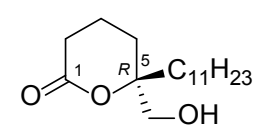

$(+)$-tanikolide $\left({ }^{1}\right)$

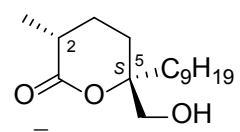

( )-malyngolide
Fig. 1. Structure of (+)-tanikolide (1) and (-)-malyngolide.

* Corresponding author. Tel.: +81-76-434-7555; fax: +81-76-4345053; e-mail: yakura@pha.u-toyama.ac.jp (T. Yakura).
In terms of potent biological activities and structural features of $(+)$-tanikolide and ( $(-)$-malyngolide, there have been a large number of reports on the total syntheses of these natural products $^{3-13}$ and their analogues. ${ }^{6 n, o}$ One of the key steps is the stereoselective construction of the quaternary carbon center at C5, which has been accomplished using several different approaches, such as aldol reactions, ${ }^{3}$ allylation of ketones such as Keck allylation, ${ }^{4}$ addition of Grignard reagents to ketones, ${ }^{5}$ epoxidation-epoxide opening, ${ }^{6} \quad$ Sharpless asymmetric dihydroxylation, $^{7}$ sigmatropic rearrangement, ${ }^{8}$ 1,3-dipolar cycloaddition of nitronate and acrolein, ${ }^{9}$ asymmetric intramolecular cyclopropanation, ${ }^{10}$ bromolactonization, ${ }^{11}$ asymmetric allylic alkylation, ${ }^{12}$ and others. ${ }^{13}$ However, most of these examples are application and demonstration of synthetic methodologies to construct a synthetically challenging quaternary carbon center. To the best of our knowledge, there is only one example of the divergent synthesis of their analogues. ${ }^{6 n, o}$

One of the most direct and powerful methods for construction of substituted cyclic ethers is tandem intramolecular oxonium ylide formation from $\alpha$-diazocarbonyl compounds under catalysis by dirhodium(II) or copper complexes and [2,3]-sigmatropic rearrangement. ${ }^{14}$ The synthetic utility of the tandem reaction has been demonstrated through the synthesis of a wide range of natural products. ${ }^{15}$ We have reported a stereoselective, coppercatalyzed oxonium ylide formation-rearrangement of $\alpha$-diazo ketone for the synthesis of the $\mathrm{C} 3-\mathrm{C} 12$ portion of laulimalide. ${ }^{16}$ We have also disclosed that a dirhodium(II)-catalyzed tandem reaction of diazoketoesters proceeds with excellent stereoselectivity ${ }^{17}$ and is applicable to the synthesis of 2-epicinatrin $\mathrm{C}_{1}$ dimethyl ester. ${ }^{18}$ As a part of our work in the 
development of oxonium ylide rearrangement and its application to the synthesis of biologically active compounds, we herein report a divergent synthesis of (+)-tanikolide and its analogues, such as 4-hydroxy- and nortanikolides. ${ }^{19}$

\section{Results and discussion}

\subsection{Synthetic strategy}

Our synthetic plan for (+)-tanikolide (1) and its analogues, 4hydroxytanikolides (2), and nortanikolide (3), is illustrated in Scheme 1. We have already reported the stereoselective dirhodium(II)-catalyzed oxonium ylide formation-[2,3]sigmatropic rearrangement of 5-allyloxy-2-diazo-3oxocarboxylate. ${ }^{17,20}$ From this observation, we can expect to stereoselectively obtain $(2 R, 5 S)$-dihydrofuranone $\mathbf{6}$, which has the same configuration at $\mathrm{C} 2$ as that of tanikolide, by the dirhodium(II)-catalyzed reaction of $(5 S)$-diazoketoester 5 . The diazoester 5 can be prepared from $(S)$-L-malic acid (4). The obtained dihydrofuranone 6 could be a key synthetic intermediate for 1-3. In addition, introduction of the alkyl side chain could be conducted at a later stage of the synthesis using cross metathesis to easily synthesize analogues with various side chains. Deoxygenation of the keto group in $\mathbf{6}$, followed by the oxidation of the resultant tetrahydrofuran-5-methanol $7\left(\mathrm{R}^{2}=\mathrm{H}\right)$, could provide tetrahydrofurfural 9. Subsequently, $N$-heterocyclic carbene (NHC)-catalyzed ring-expansion of aldehyde $\mathbf{9}$, prepared from the hydroxymethyl group in 7 , to $\delta$-lactone $\mathbf{1 1}^{21}$ and the following cross metathesis would result in the completion of the total synthesis of $\mathbf{1}$. Reduction of ketone $\mathbf{6}$ could afford the alcohol $8\left(\mathrm{R}^{2}=\mathrm{OR}^{4}\right)$, which should be converted into $\mathbf{2}$ in the same manner as that of 7 into $\mathbf{1}$. Noranalogue $\mathbf{3}$ could be synthesized from 7 by an oxidative cleavage reaction ${ }^{22}$ and cross metathesis. Consequently, in this synthesis, the C5 hydroxymethyl group plays the following important roles: construction of the $\mathrm{C} 2$ chiral center, ring-expansion from $\gamma$ lactone to $\delta$-lactone, and oxidative cleavage.

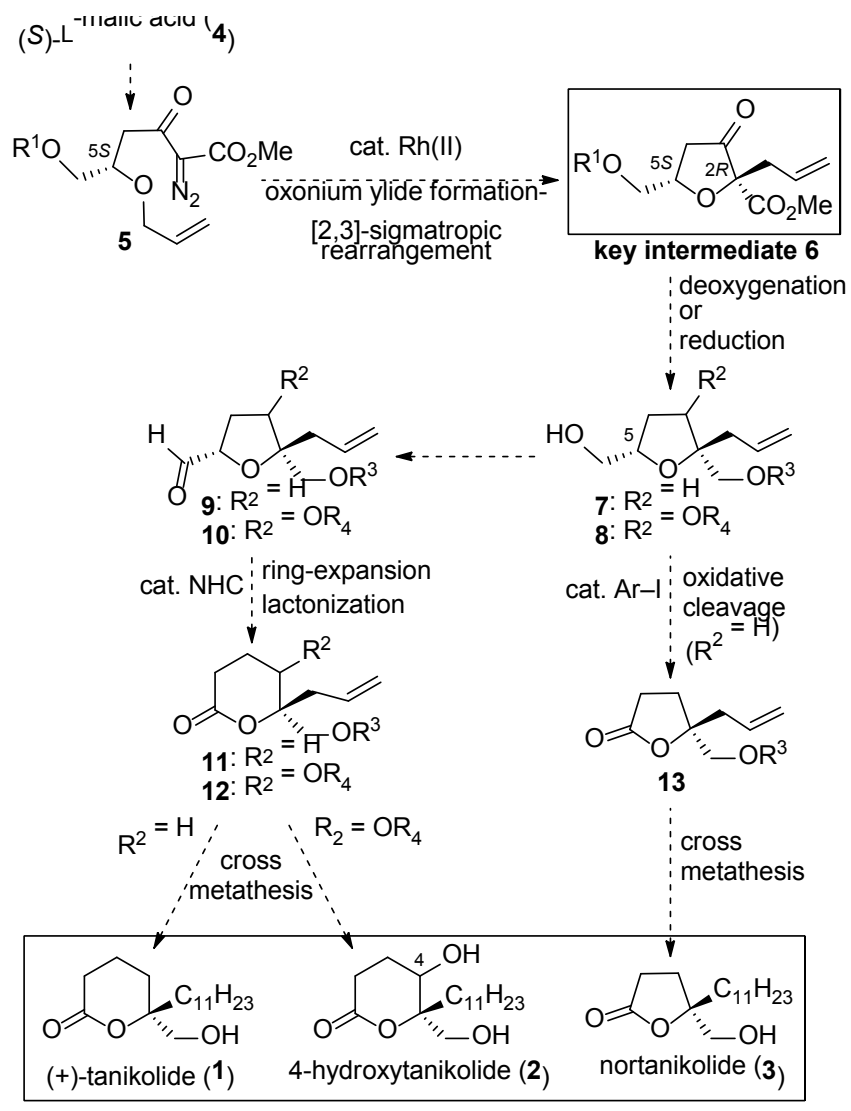

Scheme 1. Synthetic strategy for (+)-tanikolide (1) and its analogues 2 and $\mathbf{3}$.

\subsection{Total synthesis of $(+)$-tanikolide}

The synthesis of the key intermediate $\mathbf{6 a}$ from $(S)$-L-malic acid (4) was investigated as shown in Scheme 2. Conversion of $\mathbf{4}$ into silyloxyalcohol $\mathbf{1 4}$ was conducted in the usual manner. ${ }^{23}$ Allyllation of the hydroxy group in $\mathbf{1 4}$ was accomplished by the treatment with allyl bromide, silver(I) oxide, calcium sulfate, and a molecular sieve (MS) $4 \AA$ for $24 \mathrm{~h}$ in the dark, which afforded allyl ether $\mathbf{1 5}$ in $78 \%$ yield. After the reduction of ester $\mathbf{1 5}$ into aldehyde 16 with diisobutylaluminum hydride (DIBAL-H), the resultant 16 was converted to diazoketoester 5a via $\beta$-ketoester 17 by treatment of 16 with methyl diazoacetate with $\mathrm{SnCl}_{2}$ and subsequent diazo transfer from $p$-acetoamidobenzenesulfonyl azide ( $p$-ABSA) to 17. The rhodium(II)-catalyzed oxonium ylide formation-[2,3]-sigmatropic rearrangement of $\mathbf{5 a}$ yielded dihydrofuranone $\mathbf{6 a}$ with perfect stereoselectivity. The reaction of diazoketoester 5a with $3 \mathrm{~mol} \%$ of dirhodium(II) tetraacetate in refluxing dichloromethane for $5 \mathrm{~h}$ produced the corresponding dihydrofuranone $\mathbf{6 a}$ as the sole product in $93 \%$ yield. 


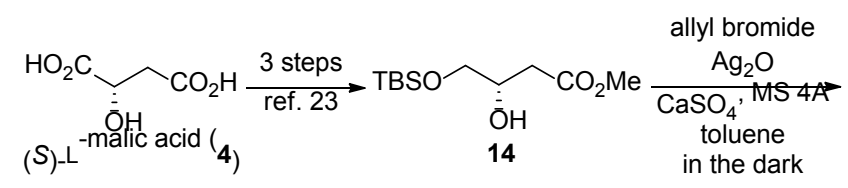

$(78 \%)$

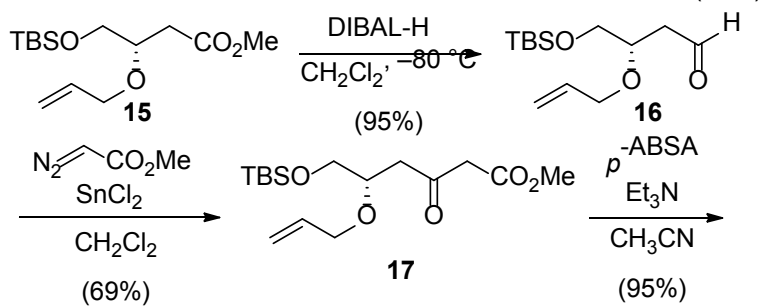

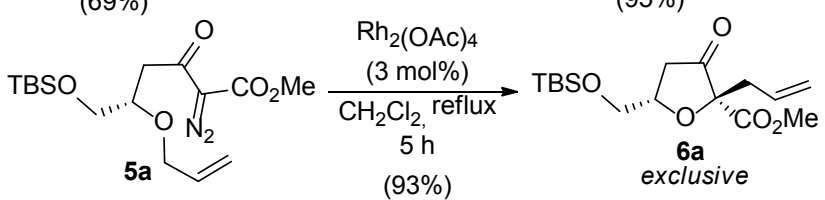

Scheme 2. Stereoselective synthesis of key intermediate $\mathbf{6 a}$

With the key synthetic intermediate 6a in hand, we next examined the deoxygenation of the keto group in dihydrofuranone 6a (Scheme 3). After conversion of 6a to alcohol $\mathbf{1 8}$ by treatment with sodium borohydride in tetrahydrofuran (THF), the reaction of $\mathbf{1 8}$ with Martin sulfurane or Burgess reagents did not proceed at all. Next, we examined the Barton-McCombie deoxygenation of alcohol 18. Treatment of $\mathbf{1 8}$ with 1,1'-thiocarbonyldiimidazole in benzene under reflux provided the corresponding thiocarbamate 19 in $95 \%$ yield. The reaction of $\mathbf{1 9}$ with tributyltin hydride in the presence of azobisisobutyronitrile (AIBN) gave a complex mixture of products. Since all the attempts to deoxygenate 3hydroxytetrahydrofuran 18 were unsuccessful, direct deoxygenation of the ketone in 6a using Wolff-Kishner-type reduction $^{24}$ was then investigated. Treatment of ketone $\mathbf{6 a}$ with tosylhydrazine in THF gave hydrazone 20a in $92 \%$ yield. According to Hutchins' modified conditions, ${ }^{24 \mathrm{~b}}$ the reaction of 20a with sodium cyanoborohydride in the presence of catalytic amounts of $p$-toluenesulfonic acid (TsOH) in $N, N$ dimethyformamide (DMF) at $130{ }^{\circ} \mathrm{C}$ provided the undesired dihydrofuran 21a in $40 \%$ yield, without any of the desired product 22. This unexpected result led us to change our synthetic plan. The cross metathesis for extension of the alkyl side chain should be conducted prior to the required hydrogenation of dihydrofuran. The reaction of $\mathbf{6 a}$ with 10 equivalent of 1 -decene in the presence of a Grubbs 2nd catalyst proceeded smoothly to afford alkene $\mathbf{2 3}$ in $88 \%$ yield. After conversion of ketone $\mathbf{2 3}$ into tosylhydrazone $\mathbf{2 0 b}$, we then examined the reduction of $\mathbf{2 0 b}$. The treatment of 20b with $\mathrm{NaBH}_{3} \mathrm{CN}$ and 0.7 equivalent of TsOH in DMF at $130{ }^{\circ} \mathrm{C}$ gave dihydrofuran $\mathbf{2 1 b}$ in only $37 \%$ yield. We assumed that the formation of $\mathbf{2 1 b}$ from $20 \mathrm{~b}$ proceeded through a Bamford-Stevens-type reaction. Therefore, we next investigated the reaction under standard Bamford-Stevens conditions. However, treatment of $\mathbf{2 0 b}$ with sodium methoxide in methanol under reflux did not afford the product $\mathbf{2 1 b}$. Thus, we examined the reaction with $\mathrm{NaBH}_{3} \mathrm{CN}$ in the absence of TsOH. Gratifyingly, the yield of 21b was increased (54\% yield). Furthermore, we found that the reaction with $\mathrm{NaBH}_{3} \mathrm{CN}$ in refluxing DMF afforded 21b in $76 \%$ yield. Catalytic hydrogenation of both cyclic and side alkenes in $\mathbf{2 1 b}$ provided tetrahydrofuran 24 in $94 \%$ yield.

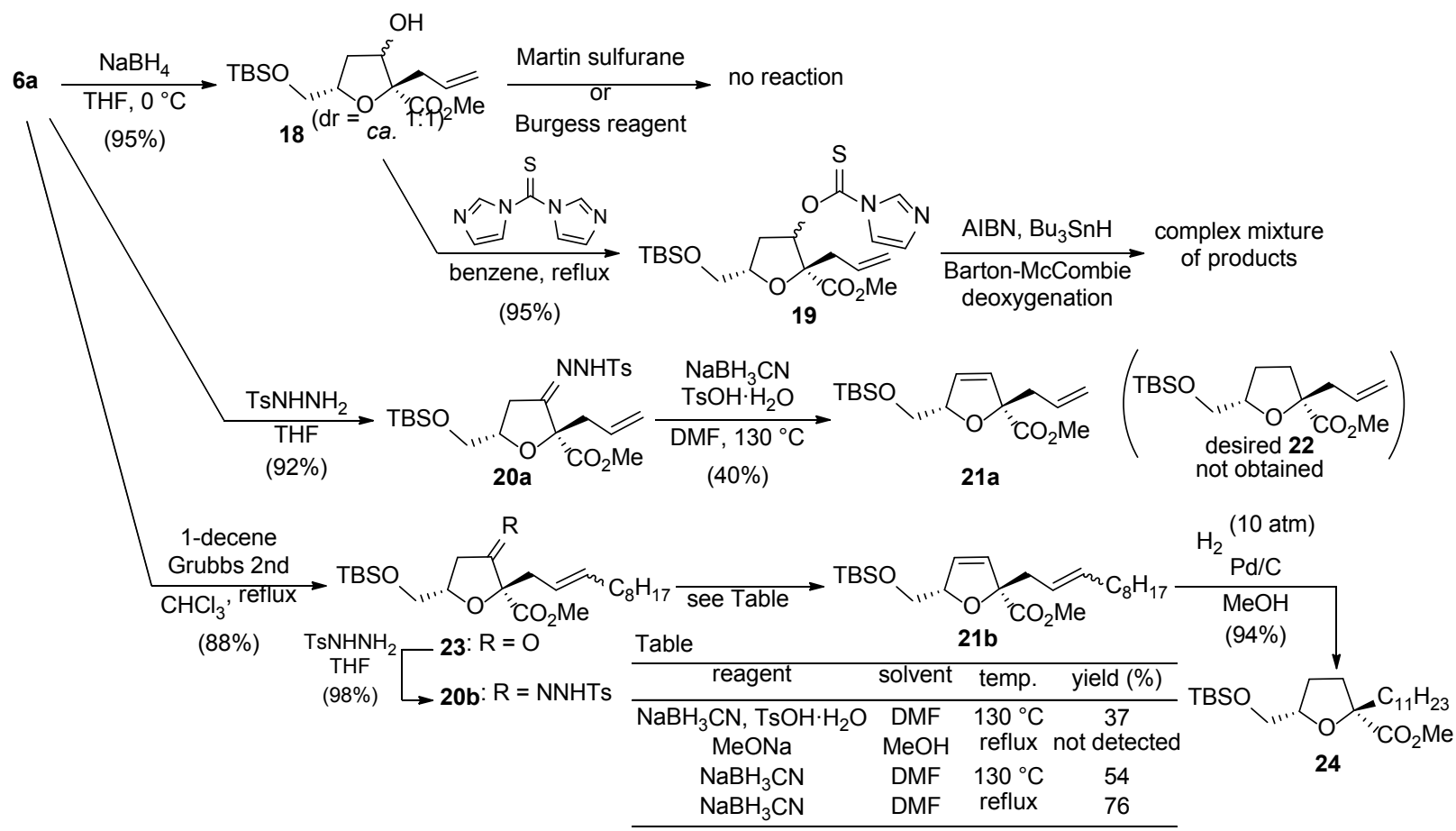

Scheme 3. Deoxygenation of dihydrofuranone $6 \mathbf{a}$. 
The stage was now set for ring-expansion from $\gamma$-lactone to $\delta$ lactone and completion of the synthesis of $(+)$-tanikolide (1, Scheme 4). Conversion of $\mathbf{2 4}$ into the precursor for NHCcatalyzed ring expansion was conducted by an uneventful fourstep transformation. Hydride reduction of methyl ester $\mathbf{2 4}$ with DIBAL-H furnished a primary alcohol 25 in $89 \%$ yield. After protection of the hydroxyl group as its methoxymethyl (MOM) ether, it was desilylated with tetrabutylammonium fluoride (TBAF), and subsequent Swern oxidation afforded aldehyde 26. We then investigate the ring expansion of tetrahydrofuran to $\delta$ lactone $\mathbf{3 0}{ }^{21}$ Treatment of $\mathbf{2 8}$ with $30 \mathrm{~mol} \%$ of imidazolium catalyst 29 in the presence of 1,8-diazabicyclo[5.4.0]undec-7-ene (DBU) in chloroform under reflux for $7 \mathrm{~h}$ gave $\delta$-lactone $\mathbf{3 0}$ in $71 \%$ yield. Finally, the MOM group in $\mathbf{3 0}$ was deprotected under acidic conditions to furnish (+)-tanikolide (1), mp 39-40 ${ }^{\circ} \mathrm{C}\left(\right.$ lit. $^{6 \mathrm{~h}}$ $\left.\operatorname{mp~} 39-41{ }^{\circ} \mathrm{C}\right),[\alpha]_{\mathrm{D}}{ }^{24}+2.2\left(c 0.65, \mathrm{CHCl}_{3}\right)\left\{\right.$ lit. $^{1}[\alpha]_{\mathrm{D}}{ }^{25}+2.3(c$ $\left.\left.0.65, \mathrm{CHCl}_{3}\right)\right\}$, in $56 \%$ yield.

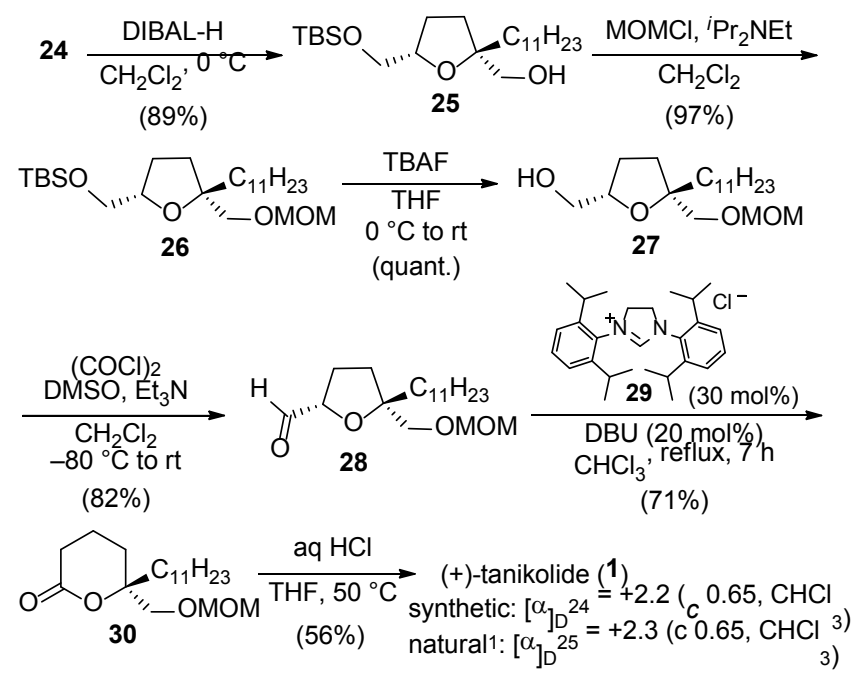

Scheme 4. Synthesis of (+)-tanikolide (1).

\subsection{Synthesis of (4S)- and (4R)-hydroxytanikolides}

Next, we synthesized (4S)- and (4R)-hydroxytanikolides (2) from the key intermediate dihydrofuranone 6a. Initially, we examined hydride reduction of $\mathbf{6 a}$ (Scheme 5). The reaction of $\mathbf{6 a}$ with lithium aluminum hydride in THF at $0{ }^{\circ} \mathrm{C}$ gave a mixture of diols (3S)- and (3R)-8a. These diastereomers could be separated by column chromatography on silica gel, and (3S)- and (3R)-8a were obtained in $52 \%$ and $37 \%$ yields, respectively. Stereochemical assignments of these diols were obtained from experiments with acetonide formation. The reaction of the major isomer of 8a with 2,2-dimethoxypropane using a catalytic amount of $\mathrm{TsOH}$ in benzene under reflux proceeded smoothly to completion within 30 min, providing acetonide 31 in 99\% yield. Conversely, the reaction of the minor isomer of $\mathbf{8 a}$ under the same conditions did not proceed, even after $24 \mathrm{~h}$. Because formation of a trans-fused [6.5]-bicyclic system is generally more difficult than that of a cis-fused one, the stereochemistries of the major and minor isomers $\mathbf{8 a}$ at $\mathrm{C} 3$ were determined to be $S$ and $R$, respectively.

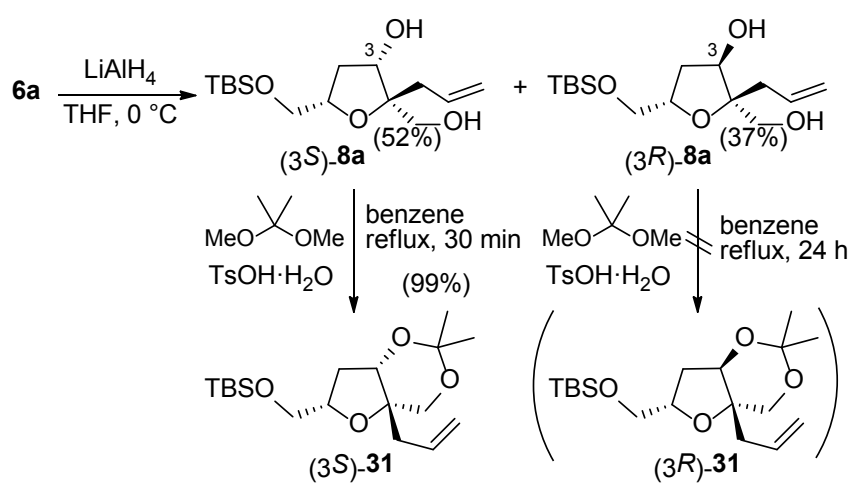

Scheme 5. Reduction of 6 a with $\mathrm{LiAlH}_{4}$.

With the acetonide (3S)-31 in hand, we then examined the synthesis of (4S)-hydroxytanikolide (2) employing NHCcatalyzed ring expansion and cross metathesis (Scheme 6). Removal of the tert-butyldimethylsilyl (TBS) protecting group in (3S)-31 with TBAF was followed by Swern oxidation to afford aldehyde (3S)-32 in 87\% yield. Conversion from (3S)-32 into $\delta$ lactone (4S)-33 was conducted under the same conditions as those for $\mathbf{2 8}$. The product (4S)-33 was obtained in $75 \%$ yield. The cross metathesis between alkene (4S)-33 and 1-decene (10 equivalent) followed by hydrogenation provided (4S)-34 in $86 \%$ yield. Hydrolysis of acetonide (4S)-34 under acidic conditions furnished (4S)-hydroxytanikolide (2) in 94\% yield.
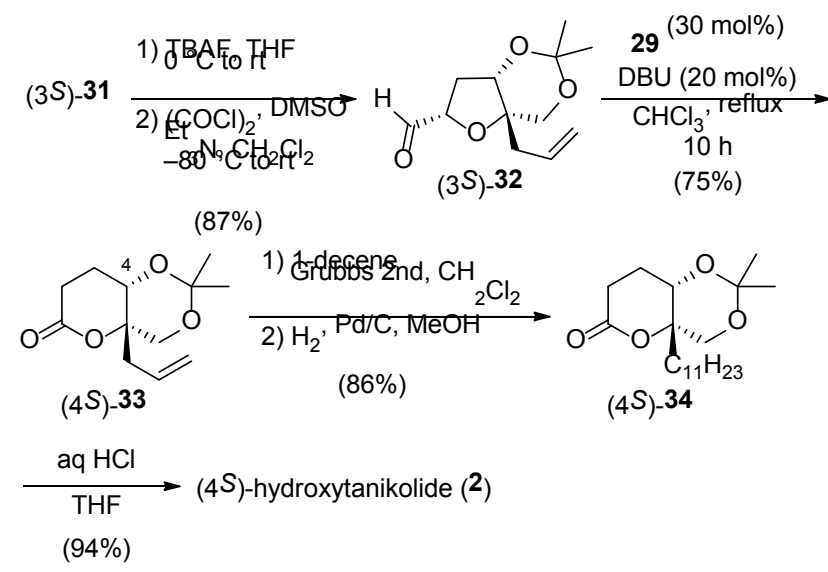

Scheme 6. Synthesis of (4S)-hydroxytanikolide (2).

Following a procedure similar to that for $(3 S)-\mathbf{8 a}$, the diol (3R)-8a was converted into (4R)-hydroxytanikolide (2, Scheme 7). Protection of the diol as the bis-MOM groups gave ( $3 R)-35$ in $84 \%$ yield. Desilylation of TBS ether (3R)-35 with TBAF and subsequent Swern oxidation provided aldehyde (3R)-36 in 73\% yield. Ring expansion of (3R)-36 using $30 \mathrm{~mol} \%$ imidazolium catalyst 29 and $20 \mathrm{~mol} \% \mathrm{DBU}$ in toluene at $80{ }^{\circ} \mathrm{C}$ proceeded to completion within $10 \mathrm{~h}$, providing $\delta$-lactone $(4 R)-37$ in $68 \%$ yield. The cross metathesis for the long side chain extension and following hydrogenation furnished $(4 R)-38$ in $66 \%$ yield. The bis-MOM groups in (4R)-38 were deprotected under acidic conditions to afford (4R)-hydroxytanikolide (2) in $90 \%$ yield. 


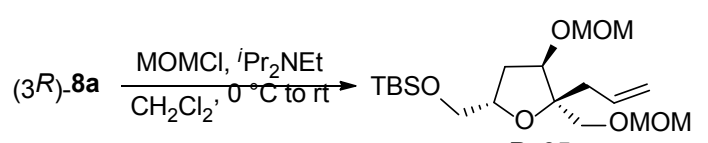

$(84 \%)$ $(3 R)-35$
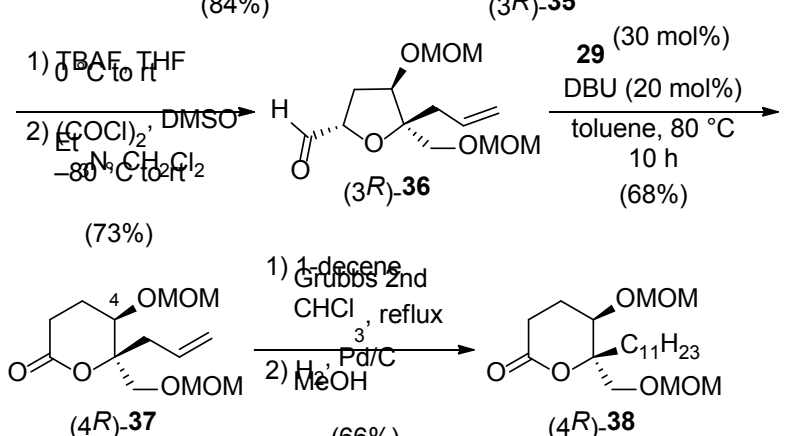

$(4 R)-37$

$(66 \%)$

$(4 R)-38$

$\underset{\mathrm{THF}, 50{ }^{\circ} \mathrm{C}}{\stackrel{\text { aq } \mathrm{HCl}}{\longrightarrow}}(4 R)$-hydroxytanikolide (2)

$(90 \%)$

Scheme 7. Synthesis of (4R)-hydroxytanikolide (2).

\subsection{Synthesis of nortanikolide}

Finally, the synthesis of nortanikolide (3) from the tetrahydrofuran $\mathbf{2 5}$, which was used as a synthetic intermediate for (+)-tanikolide (1), was investigated (Scheme 8). After protection of the hydroxyl group in $\mathbf{2 5}$ as its benzyl (Bn) ether, deprotection of the TBS ether with TBAF afforded alcohol $\mathbf{4 0}$ in $84 \%$ yield. Then, we examined the oxidative cleavage of tetrahydrofuran-5-methanol $\mathbf{4 0}$ to $\gamma$-lactone. In our previous report on the synthesis of 2-epi-cinatrin $\mathrm{C}_{1}$ dimethyl ester, we employed the oxidative cleavage of tetrahydrofuran-5-methanol to $\gamma$-lactone with an excess amount of pyridinium dichromate and acetic anhydride in $\mathrm{CH}_{2} \mathrm{Cl}_{2}-\mathrm{DMF}$ under reflux. ${ }^{18}$ Although the $\gamma$ lactone was obtained in good yield, the reaction was carried out under harsh conditions, such as the use of highly toxic hexavalent chromium oxidants and heating at high temperature. To avoid these drawbacks, we recently developed an environmentally benign method for oxidative cleavage using a 2-iodobenzamide catalyst $41 .^{22}$ Therefore, we examined the oxidative cleavage under our original conditions. The reaction of $\mathbf{4 0}$ with $30 \mathrm{~mol} \%$ 41 and 5 equivalent of Oxone in DMF at room temperature provided the corresponding $\gamma$-lactone $\mathbf{4 2}$ in $65 \%$ yield; however, unexpectedly, benzoate $\mathbf{4 3}$ was obtained in $12 \%$ yield. This result indicates that the oxidation at the benzylic position of $\mathbf{4 0}$ proceeded in part to give $\mathbf{4 3}^{25}$ To prevent this undesired oxidation, we explored the use of an antioxidant. As expected, the reaction of $\mathbf{4 0}$ using $30 \quad \mathrm{~mol} \%$ 2,6-di-tert-butyl-4hydroxytoluene (BHT) as an antioxidant suppressed the formation of benzoate $\mathbf{4 3}$ and afforded $\mathbf{4 2}$ in $83 \%$ yield. Finally, hydrogenolysis of the benzyl ether in the presence of Pearlman's catalyst $\left(\mathrm{Pd}(\mathrm{OH})_{2} / \mathrm{C}\right)$ in methanol furnished nortanikolide (3) in $94 \%$ yield.

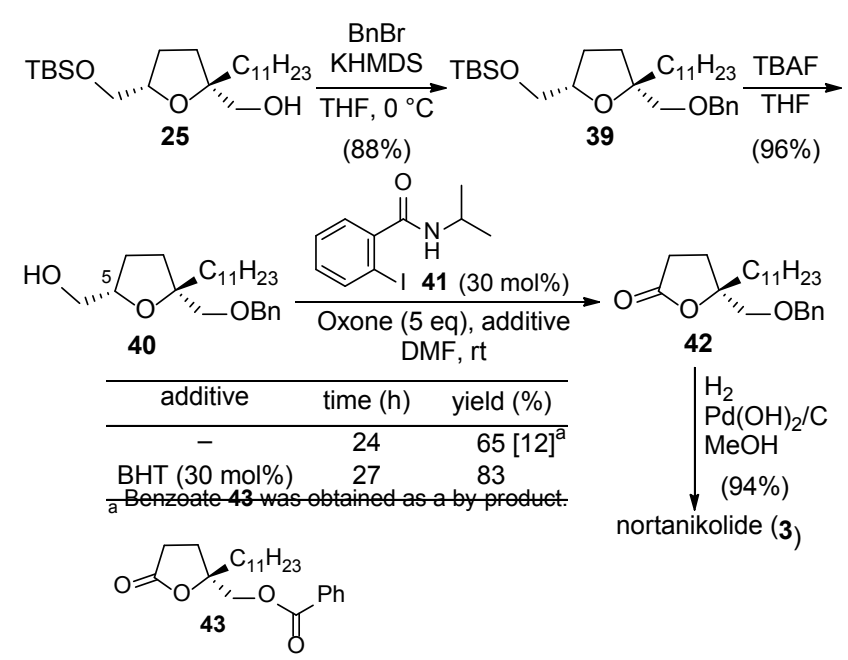

Scheme 8. Synthesis of nortanikolide (3).

\section{Conclusion}

The divergent synthesis of (+)-tanikolide, (4S)- and (4R)hydroxytanikolides, and nortanikolide from the key intermediate franone was achieved. The key steps involved a rhodium(II)catalyzed oxonium ylide formation-[2,3]-sigmatropic rearrangement, an NHC-catalyzed ring expansion of tetrahydrofurfural to $\delta$-lactone, or an oxidative cleavage of tetrahydrofuran-5-methanol to $\gamma$-lactone using a 2 -iodobenzamide catalyst. As the quaternary chiral center can be easily and stereoselectively constructed, the long side chain can be introduced at a later stage in the synthesis, and $\delta$ - and $\gamma$-lactone rings can be formed from the common intermediate, the present protocol provides high flexibility for the synthesis of a variety of analogues.

\section{Experimental section}

\subsection{General}

Melting points were determined using a Yanaco micro melting point apparatus and are uncorrected. Optical rotations were determined using a JASCO P-2100 polarimeter. Infrared (IR) spectra were recorded on a JASCO FT/IR-460 Plus spectrophotometer and absorbance bands are reported in wavenumber $\left(\mathrm{cm}^{-1}\right)$. ${ }^{1} \mathrm{H}$ NMR spectra were recorded on JEOL JNM-ECX400P (400 MHz) spectrometer. Chemical shifts are reported relative to internal standard (tetramethylsilane at $\delta_{\mathrm{H}} 0.00$ or $\mathrm{CDCl}_{3}$ at $\delta_{\mathrm{H}}$ 7.26). Data are presented as follows: chemical shift $(\delta, \mathrm{ppm})$, multiplicity $(\mathrm{s}=$ singlet, $\mathrm{d}=$ doublet, $\mathrm{t}=$ triplet, quint $=$ quintet, $\mathrm{m}=$ multiplet, $\mathrm{br}=$ broad $)$, coupling constant and integration. ${ }^{13} \mathrm{C}$ NMR spectra were recorded on JEOL JNMECX400P (100 MHz) spectrometer. The following internal reference was used $\left(\mathrm{CDCl}_{3}\right.$ at $\left.\delta 77.0\right)$. All ${ }^{13} \mathrm{C}$ NMR spectra were determined with complete proton decoupling. High-resolution mass spectra (HRMS) were determined with JEOL JMS-GCmate II and JEOL JMS-AX505HAD instruments. Column chromatography was performed on Silica Gel $60 \mathrm{PF}_{254}$ (Nacalai Tesque) and Kanto silica gel $60 \mathrm{~N}$ (63-210 mesh) under pressure. Analytical thin layer chromatography (TLC) was carried out on Merck Kieselgel $60 \mathrm{~F}_{254}$ plates. Visualization was accomplished with UV light and phosphomolybdic acid stain solution followed by heating.

4.2. Synthesis of (+)-tanikolide (1) 
4.2.1. (S)-Methyl butyldimethylsilyloxy)butanoate $(\mathbf{1 5})^{15 j}$

3-allyloxy-4-(tert-

Allyl bromide ( $3.3 \mathrm{ml}, 37.7 \mathrm{mmol}), \mathrm{Ag}_{2} \mathrm{O}$ (4.36 g, $\left.18.8 \mathrm{mmol}\right)$, and $\mathrm{CaSO}_{4}(3.42 \mathrm{~g}, 25.1 \mathrm{mmol})$ were added to a solution of $(S)$ methyl 4-(tert-butylsilyloxy)-3-hydroxybutanoate $(\mathbf{1 4})^{23}(2.34 \mathrm{~g}$, $9.42 \mathrm{mmol})$ and MS4A (2.34 g) in toluene $(38 \mathrm{ml})$ at room temperature under $\mathrm{N}_{2}$ in the dark. After stirring for 1 day, $\mathrm{Ag}_{2} \mathrm{O}$ (4.36 g, $18.8 \mathrm{mmol})$ and $\mathrm{CaSO}_{4}(3.42 \mathrm{~g}, 25.1 \mathrm{mmol})$ were added again to the reaction mixture and the mixture was stirred for 1 day. After further addition of $\mathrm{Ag}_{2} \mathrm{O}(4.36 \mathrm{~g}, 18.8 \mathrm{mmol})$ and $\mathrm{CaSO}_{4}(3.42 \mathrm{~g}, 25.1 \mathrm{mmol})$, the mixture was stirring for one more day. The reaction mixture was filtered through the pad of Celite. The filtrate was concentrated in vacuo, and the residue was purified by column chromatography (silica gel, 4\% EtOAc in hexane) to provide $15(2.13 \mathrm{~g}, 78 \%)$ as a colorless oil: $[\alpha]_{\mathrm{D}}{ }^{24}-$ 22.9 (c 1.0, $\mathrm{CHCl}_{3}$ ); IR (neat, $\mathrm{cm}^{-1}$ ) v 2954, 2930, 2886, 2858, $1742,1084,1006,838,778,669 ;{ }^{1} \mathrm{H}$ NMR $\left(400 \mathrm{MHz}, \mathrm{CDCl}_{3}\right) \delta$ $5.88(\mathrm{ddt}, J=17.0,10.4,6.0 \mathrm{~Hz}, 1 \mathrm{H}), 5.25(\mathrm{dd}, J=17.0,1.4 \mathrm{~Hz}$, $1 \mathrm{H}), 5.15(\mathrm{dd}, J=10.4,1.4 \mathrm{~Hz}, 1 \mathrm{H}), 4.15-4.04(\mathrm{~m}, 2 \mathrm{H}), 3.72$ $(\mathrm{dd}, J=10.6,8.0 \mathrm{~Hz}, 1 \mathrm{H}), 3.69(\mathrm{~s}, 3 \mathrm{H}), 3.55(\mathrm{dd}, J=10.6,5.4 \mathrm{~Hz}$ $1 \mathrm{H}), 2.61(\mathrm{dd}, J=15.6,5.4 \mathrm{~Hz}, 1 \mathrm{H}), 2.49$ (dd, $J=15.6,8.0 \mathrm{~Hz}$, $1 \mathrm{H}), 0.89$ (s, 9H), $0.06(\mathrm{~s}, 6 \mathrm{H}) ;{ }^{13} \mathrm{C} \mathrm{NMR}\left(100 \mathrm{MHz}, \mathrm{CDCl}_{3}\right) \delta$ $172.2,135.0,116.8,76.4,71.5,64.7,51.6,37.3,25.9,18.3,-5.4$.

\subsection{2. (S)-3-Allyloxy-4-(tert-butyldimethylsilyloxy)butanal (16)}

DIBAL-H (1.0 M in hexane, $12.7 \mathrm{~mL}, 12.7 \mathrm{mmol})$ was added dropwise to a solution of methyl ester $15(3.05 \mathrm{~g}, 10.6 \mathrm{mmol})$ in $\mathrm{CH}_{2} \mathrm{Cl}_{2}(106 \mathrm{~mL})$ at $-78^{\circ} \mathrm{C}$ under $\mathrm{N}_{2}$. After stirring at $-78^{\circ} \mathrm{C}$ for $30 \mathrm{~min}$, the reaction was quenched by the addition of $\mathrm{MeOH}(25$ $\mathrm{mL})$ and saturated aqueous $\mathrm{NH}_{4} \mathrm{Cl}(12.7 \mathrm{~mL})$ and then allowed to warm to room temperature. The mixture was dried over anhydrous $\mathrm{Na}_{2} \mathrm{SO}_{4}$ and filtered through a pad of Celite. The filtrate was concentrated in vacuo and the residue was purified by column chromatography (silica gel, 5\% EtOAc in hexane) to provide $16(2.59 \mathrm{~g}, 95 \%)$ as a colorless oil: $[\alpha]_{\mathrm{D}}{ }^{24}-25.3$ (c 1.0, $\mathrm{CHCl}_{3}$ ); IR (neat, $\mathrm{cm}^{-1}$ ) $\vee 2954,2929,2857,1730,1255,1094$, 838, 778; ${ }^{1} \mathrm{H}$ NMR $\left(400 \mathrm{MHz}, \mathrm{CDCl}_{3}\right) \delta 9.81(\mathrm{t}, J=2.4 \mathrm{~Hz}, 1 \mathrm{H})$, 5.89 (ddt, $J=17.0,10.4,5.6 \mathrm{~Hz}, 1 \mathrm{H}$ ), 5.27 (ddd, $J=17.0,3.2$, $1.4 \mathrm{~Hz}, 1 \mathrm{H}$ ), 5.17 (ddd, $J=10.4,3.2,1.4 \mathrm{~Hz}, 1 \mathrm{H}$ ), 4.13 (ddt, $J=$ 12.7, 5.6, $1.4 \mathrm{~Hz}, 1 \mathrm{H}), 4.06$ (ddt, $J=12.7,5.6,1.4 \mathrm{~Hz}, 1 \mathrm{H}), 3.93$ (quint, $J=5.8 \mathrm{~Hz}, 1 \mathrm{H}), 3.74(\mathrm{dd}, J=10.4,5.0 \mathrm{~Hz}, 1 \mathrm{H}), 3.59$ (dd, $J=10.4,6.2 \mathrm{~Hz}, 1 \mathrm{H}), 2.64-2.62(\mathrm{~m}, 2 \mathrm{H}), 0.89$ (s, 9H), 0.06 (s, $6 \mathrm{H}) ;{ }^{13} \mathrm{C} \mathrm{NMR}\left(100 \mathrm{MHz}, \mathrm{CDCl}_{3}\right) \delta 201.1,134.7,117.1,74.9$, $71.1,64.6,46.3,25.8,18.3,-5.5$.

4.2.3. (S)-Methyl 5-allyloxy-6-tert-butyldimethylsilyloxy-3oxohexanoate (17)

A solution of the methyl diazoacetate $(329 \mathrm{mg}, 3.29 \mathrm{mmol})$ in $\mathrm{CH}_{2} \mathrm{Cl}_{2}(9 \mathrm{~mL})$ and a solution of aldehyde $\mathbf{1 6}(567 \mathrm{mg}, 2.19$ mmol) in $\mathrm{CH}_{2} \mathrm{Cl}_{2}(15 \mathrm{~mL})$ were added to a suspension of $\mathrm{SnCl}_{2}$ $(83 \mathrm{mg}, 0.44 \mathrm{mmol})$ in $\mathrm{CH}_{2} \mathrm{Cl}_{2}(10 \mathrm{~mL})$ over $20 \mathrm{~min}$ at room temperature under $\mathrm{N}_{2}$. After stirring for $5.5 \mathrm{~h}$, the reaction mixture was evaporated and the residue was purified by column chromatography (silica gel, 7\% EtOAc in hexane) to provide $\mathbf{1 7}$ $(501 \mathrm{mg}, 69 \%)$ as a pale yellow oil: $[\alpha]_{\mathrm{D}}{ }^{25}-24.8\left(\mathrm{c} 1.0, \mathrm{CHCl}_{3}\right)$; IR $\left(\right.$ neat, $\left.\mathrm{cm}^{-1}\right) \vee 2954,2929,2885,2858,1751,1720,1322$, $1255,1117,838,778 ;{ }^{1} \mathrm{H}$ NMR $\left(400 \mathrm{MHz}, \mathrm{CDCl}_{3}\right) \delta 5.87$ (ddt, $J$ $=22.6,10.6,5.6 \mathrm{~Hz}, 1 \mathrm{H}), 5.24(\mathrm{dd}, J=17.8,1.4 \mathrm{~Hz}, 1 \mathrm{H}), 5.15$ $(\mathrm{dd}, J=10.6,1.0 \mathrm{~Hz}, 1 \mathrm{H}) 4.11(\mathrm{dd}, J=12.6,5.6 \mathrm{~Hz}, 1 \mathrm{H}), 4.03$ (dd, $J=12.6,5.6 \mathrm{~Hz}, 1 \mathrm{H}), 3.88$ (quint, $J=6.0 \mathrm{~Hz}, 1 \mathrm{H}$ ), 3.74 (s, $3 \mathrm{H}), 3.68$ (dd, $J=10.4,4.8 \mathrm{~Hz}, 1 \mathrm{H}), 3.55(\mathrm{dd}, J=10.4,6.0 \mathrm{~Hz}$, $1 \mathrm{H}), 3.52$ (s, 2H), 2.75 (dd, $J=6.0,1.3 \mathrm{~Hz}, 2 \mathrm{H}), 0.89$ (s, 9H), $0.06(\mathrm{~s}, 6 \mathrm{H}) ;{ }^{13} \mathrm{C}$ NMR $\left(100 \mathrm{MHz}, \mathrm{CDCl}_{3}\right) \delta 201.4,167.5,134.8$, 117.0, 75.8, 71.4, 64.5, 52.3, 50.1, 45.4, 25.9, 18.3, -5.5; HRMS (EI) $m / z$ calcd for $\mathrm{C}_{16} \mathrm{H}_{30} \mathrm{O}_{5} \mathrm{Si}(\mathrm{M})^{+} 330.1863$, found 330.1854 .
4.2.4. (S)-Methyl 5-allyloxy-6-tert-butyldimethylsilyloxy-2-diazo3-oxohexanoate $(\mathbf{5 a})$

A solution of $p$-ABSA $(1.88 \mathrm{~g}, 7.84 \mathrm{mmol})$ in $\mathrm{CH}_{3} \mathrm{CN}(12$ $\mathrm{mL})$ and $\mathrm{Et}_{3} \mathrm{~N}(1.8 \mathrm{ml}, 13.1 \mathrm{mmol})$ was added dropwise to a solution of $\beta$-keto ester 17 (2.16 g, $6.53 \mathrm{mmol})$ in $\mathrm{CH}_{3} \mathrm{CN}(70$ $\mathrm{mL}$ ) at $0{ }^{\circ} \mathrm{C}$ under $\mathrm{N}_{2}$. After stirring at room temperature for $5.5 \mathrm{~h}$, the reaction mixture was evaporated. The residue was dissolved in ether $(80 \mathrm{~mL})$, and washed with $10 \%$ aqueous $\mathrm{KOH}(2 \times 30$ $\mathrm{mL})$ water $(20 \mathrm{~mL})$ and brine $(20 \mathrm{~mL})$, and dried over anhydrous $\mathrm{MgSO}_{4}$. The filtrate was concentrated in vacuo, and the residue was purified by column chromatography (silica gel, 7\% EtOAc in hexane) to provide $5 \mathrm{a}(2.22 \mathrm{~g}, 95 \%)$ as a yellow oil: $[\alpha]_{\mathrm{D}}{ }^{24}-13.1$ $\left(c\right.$ 1.0, $\mathrm{CHCl}_{3}$ ); IR (neat, $\mathrm{cm}^{-1}$ ) v 2955, 2929, 2857, 2135, 1726, $1658,1438,1314,1255,1200,1123,838,778 ;{ }^{1} \mathrm{H}$ NMR $(400$ $\left.\mathrm{MHz}, \mathrm{CDCl}_{3}\right) \delta 5.89$ (ddd, $\left.J=22.6,10.8,5.6 \mathrm{~Hz}, 1 \mathrm{H}\right), 5.24(\mathrm{dd}$, $J=17.4,1.4 \mathrm{~Hz}, 1 \mathrm{H}), 5.13$ (br d, $J=10.8 \mathrm{~Hz}, 1 \mathrm{H}), 4.99(\mathrm{~m}, 1 \mathrm{H})$, $4.16-4.06(\mathrm{~m}, 2 \mathrm{H}), 3.83(\mathrm{~s}, 3 \mathrm{H}), 3.71(\mathrm{dd}, J=10.4,5.6 \mathrm{~Hz}, 1 \mathrm{H})$, 3.17 (dd, $J=16.4,8.0 \mathrm{~Hz}, 1 \mathrm{H}), 3.01$ (dd, $J=16.4,4.6 \mathrm{~Hz}, 1 \mathrm{H}$ ), 0.89 (s, 9H), 0.06 (s, 6H); ${ }^{13} \mathrm{C}$ NMR $\left(100 \mathrm{MHz}, \mathrm{CDCl}_{3}\right) \delta 190.5$, 161.7, 135.2, 116.6, 75.8, 71.5, 65.1, 52.2, 42.6, 25.8, 18.3, -5.4; HRMS (EI) $m / z$ calcd for $\mathrm{C}_{16} \mathrm{H}_{28} \mathrm{~N}_{2} \mathrm{O}_{5} \mathrm{Si}(\mathrm{M})^{+} 356.1768$, found 356.1793 .

4.2.5.

$(2 R, 5 S)-$ Methyl

2-allyl-5-(tert-

butyldimethylsilyloxy)methyl-3-oxotetrahydrofuran-2-

carboxylate (6a)

A solution of diazoketoester $5 \mathrm{a}(1.27 \mathrm{~g}, 3.56 \mathrm{mmol})$ in $\mathrm{CH}_{2} \mathrm{Cl}_{2}$ $(17 \mathrm{~mL})$ was added dropwise to a solution of $\mathrm{Rh}_{2}(\mathrm{OAc})_{4}(47 \mathrm{mg}$, $0.11 \mathrm{mmol}, 3 \mathrm{~mol} \%)$ in $\mathrm{CH}_{2} \mathrm{Cl}_{2}(100 \mathrm{~mL})$ at room temperature under $\mathrm{N}_{2}$. After refluxing for $5 \mathrm{~h}$, the reaction mixture was evaporated and the residue was purified by column chromatography (silica gel, 10\% EtOAc in hexane) to provide $\mathbf{6 a}$ $(1.08 \mathrm{~g}, 93 \%)$ as a colorless oil: $[\alpha]_{\mathrm{D}}{ }^{24}+5.5\left(c 1.0, \mathrm{CHCl}_{3}\right)$; IR $\left(\right.$ neat, $\left.\mathrm{cm}^{-1}\right) \vee 2954,2929,2858,1769,1748,1435,1174,1109$, 838, 779; ${ }^{1} \mathrm{H}$ NMR $\left(400 \mathrm{MHz}, \mathrm{CDCl}_{3}\right) \delta 5.74$ (ddt, $J=16.9,10.1$, $7.3 \mathrm{~Hz}, 1 \mathrm{H}), 5.20-5.13(\mathrm{~m}, 2 \mathrm{H}), 4.47(\mathrm{~m}, 1 \mathrm{H}), 3.87(\mathrm{dd}, J=10.8$, $4.6 \mathrm{~Hz}, 1 \mathrm{H}), 3.80(\mathrm{dd}, J=10.8,3.6 \mathrm{~Hz}, 1 \mathrm{H}), 3.75$ (s, 3H), 2.71 (ddt, $J=13.8,7.3,0.9 \mathrm{~Hz}, 1 \mathrm{H}), 2.65$ (dd, $J=18.1,6.4 \mathrm{~Hz}, 1 \mathrm{H})$, 2.60 (ddt, $J=13.8,7.3,0.9 \mathrm{~Hz}, 1 \mathrm{H}), 2.56(\mathrm{dd}, J=18.1,7.3 \mathrm{~Hz}$, $1 \mathrm{H}), 0.88(\mathrm{~s}, 9 \mathrm{H}), 0.06(\mathrm{~s}, 6 \mathrm{H}) ;{ }^{13} \mathrm{C} \mathrm{NMR}\left(100 \mathrm{MHz}, \mathrm{CDCl}_{3}\right) \delta$ 208.9, 168.2, 130.8, 120.3, 86.4, 76.1, 65.4, 52.8, 39.7, 38.5, 25.8, $18.3,-5.5,-5.6$; HRMS (EI) $\mathrm{m} / z$ calcd for $\mathrm{C}_{16} \mathrm{H}_{28} \mathrm{O}_{5} \mathrm{Si}(\mathrm{M})^{+}$ 328.1706 , found 328.1705 .

4.2.6. (2R,5S)-Methyl 5-(tert-butyldimethylsilyloxy)methyl-3-(4toluenesulfonyl) hydrazinylidene-2-allyltetrahydrofuran-2-

carboxylate (20a)

Tosylhydrazine (109 $\mathrm{mg}, 0.58 \mathrm{mmol})$ was added to a solution of ketone $6 \mathrm{a}(174 \mathrm{mg}, 0.53 \mathrm{mmol})$ in THF $(0.9 \mathrm{~mL})$ at room temperature under $\mathrm{N}_{2}$. After stirring at room temperature for $24 \mathrm{~h}$, solvent was removed in vacuo and the residue was purified by column chromatography (silica gel, 20\% EtOAc in hexane) to provide 20a (242 $\mathrm{mg}, 92 \%)$ as a colorless oil: $[\alpha]_{\mathrm{D}}{ }^{19}-4.4(c 1.0$, $\mathrm{CHCl}_{3}$ ); IR (neat, $\mathrm{cm}^{-1}$ ) v 3209, 2953, 2928, 1745, 1435, 1411, 1347, 1254, 1169, 923, 838, 778; ${ }^{1} \mathrm{H}$ NMR (400 MHz, $\mathrm{CDCl}_{3}$ ) $\delta 7.83(\mathrm{dt}, J=8.7,1.8 \mathrm{~Hz}, 2 \mathrm{H}), 7.33(\mathrm{~s}, 2 \mathrm{H}), 7.31(\mathrm{~s}, 1 \mathrm{H}), 5.57$ (ddt, $J=17.2,10.1,6.9 \mathrm{~Hz}, 1 \mathrm{H}), 4.98(\mathrm{dt}, J=10.1,1.4 \mathrm{~Hz}, 1 \mathrm{H})$, 4.91 (ddd, $J=17.2,3.2,1.4 \mathrm{~Hz}, 1 \mathrm{H}$ ), 4.30 (ddd, $J=6.9,6.8,4.6$ $\mathrm{Hz}, 1 \mathrm{H}), 3.77$ (dd, $J=10.3,4.6 \mathrm{~Hz}, 1 \mathrm{H}), 3.611(\mathrm{~s}, 3 \mathrm{H}), 3.609$ (dd, $J=10.3,6.9 \mathrm{~Hz}, 1 \mathrm{H}), 2.75(\mathrm{dd}, J=14.2,6.9 \mathrm{~Hz}, 1 \mathrm{H}), 2.60-2.53$ $(\mathrm{m}, 2 \mathrm{H}), 2.46(\mathrm{dd}, J=17.4,6.9 \mathrm{~Hz}, 1 \mathrm{H}), 2.43(\mathrm{~s}, 3 \mathrm{H}), 0.84(\mathrm{~s}$, 9H), 0.02 (s, 3H), 0.01 (s, 3H); ${ }^{13} \mathrm{C}$ NMR (100 MHz, $\mathrm{CDCl}_{3}$ ) $\delta 159.7,144.3,135.1,131.4,129.5,128.1,119.7,86.2,78.7,77.2$, $64.9,52.6,40.6,30.2,25.8,21.6,18.2,-5.4,-5.5$; HRMS (EI) $\mathrm{m} / \mathrm{z}$ calcd for $\mathrm{C}_{23} \mathrm{H}_{36} \mathrm{~N}_{2} \mathrm{O}_{6} \mathrm{SiS}(\mathrm{M})^{+} 496.2063$, found 496.2083. 
4.2.7. (2R,5S)-Methyl 5-(tert-butyldimethylsilyloxy)methyl-2,5dihydro-2-allylfuran-2-carboxylate (21a)

Sodium cyanoborohydride $(30 \mathrm{mg}, 0.48 \mathrm{mmol})$ and $\mathrm{TsOH} \cdot \mathrm{H}_{2} \mathrm{O}(7 \mathrm{mg}, 0.04 \mathrm{mmol})$ were added to a solution of tosylhydrazone $20 \mathrm{a}(60 \mathrm{mg}, 0.12 \mathrm{mmol})$ in DMF $(2.4 \mathrm{~mL})$ at room temperature. After refluxing for $8 \mathrm{~h}$, the reaction mixture was diluted with EtOAc $(30 \mathrm{~mL})$ and washed with water $(20 \mathrm{~mL})$ and brine $(20 \mathrm{~mL})$, and dried over anhydrous $\mathrm{MgSO}_{4}$. The filtrate was concentrated in vacuo, and the residue was purified by column chromatography (silica gel, 3\% EtOAc in hexane) to provide $21 \mathrm{a}(14 \mathrm{mg}, 40 \%)$ as a colorless oil: $[\alpha]_{\mathrm{D}}{ }^{25}-79.9(c 1.0$, $\mathrm{CHCl}_{3}$ ); IR (neat, $\mathrm{cm}^{-1}$ ) v 2953, 2929, 2857, 1759, 1735, 1256, $1128,1097,1074,917,838,778 ;{ }^{1} \mathrm{H}$ NMR $\left(400 \mathrm{MHz}, \mathrm{CDCl}_{3}\right) \delta$ $6.03(\mathrm{dd}, J=6.0,1.4 \mathrm{~Hz}, 1 \mathrm{H}), 5.88(\mathrm{dd}, J=6.0,2.3 \mathrm{~Hz}, 1 \mathrm{H})$, $5.75(\mathrm{~m}, 1 \mathrm{H}), 5.13-5.07(\mathrm{~m}, 2 \mathrm{H}), 4.87$ (ddd, $J=6.9,5.0,2.5 \mathrm{~Hz}$, $1 \mathrm{H}), 3.83(\mathrm{dd}, J=9.8,5.0 \mathrm{~Hz}, 1 \mathrm{H}), 3.72(\mathrm{~s}, 3 \mathrm{H}), 3.55(\mathrm{dd}, J=9.8$, $6.9 \mathrm{~Hz}, 1 \mathrm{H}), 2.65$ (ddt, $J=14.1,7.3,1.4 \mathrm{~Hz}, 1 \mathrm{H}), 2.54$ (ddt, $J=$ 14.1, 7.3, $1.4 \mathrm{~Hz}, 1 \mathrm{H}), 0.88$ (s, 9H), 0.05 (s, 3H), 0.04 (s, 3H); ${ }^{13} \mathrm{C}$ NMR $\left(100 \mathrm{MHz}, \mathrm{CDCl}_{3}\right) \delta 172.7,132.0,130.7,129.1,118.8$, 93.2, 87.9, 65.9, 52.2, 42.4, 25.8, 18.3, -5.4, -5.5; HRMS (FAB) $\mathrm{m} / \mathrm{z}$ calcd for $\mathrm{C}_{16} \mathrm{H}_{29} \mathrm{O}_{4} \mathrm{Si}(\mathrm{M}+\mathrm{H})^{+} 313.1835$, found 313.1840

4.2.8. (2R,5S)-Methyl 5-(tert-butyldimethylsilyloxy)methyl-2-(2undecen-1-yl)-3-oxotetrahydrofuran-2-carboxylate (23)

Grubbs 2 nd catalyst ( $4.7 \mathrm{mg}, 0.006 \mathrm{mmol}, 1 \mathrm{~mol} \%$ ) was added to the solution of $6 \mathbf{a}(181 \mathrm{mg}, 0.55 \mathrm{mmol})$ and 1-decene $(1.0 \mathrm{~mL}$, $5.51 \mathrm{mmol})$ in $\mathrm{CHCl}_{3}(5.5 \mathrm{ml})$ at room temperature under $\mathrm{N}_{2}$. After refluxing for $3 \mathrm{~h}$, Grubbs $2 \mathrm{nd}$ catalyst $(4.7 \mathrm{mg}, 0.006 \mathrm{mmol}$, $1 \mathrm{~mol} \%$ ) was added again to the reaction mixture and the mixture was refluxed for $3.5 \mathrm{~h}$. Solvent was removed in vacuo, and the residue was purified by column chromatography (silica gel, 5\% EtOAc in hexane) to provide $\mathbf{2 3}$ (214 $\mathrm{mg}, 88 \%$ ) as a pale yellow oil: $[\alpha]_{\mathrm{D}}{ }^{24}+11.3\left(c\right.$ 1.0, $\left.\mathrm{CHCl}_{3}\right)$; IR (neat, $\left.\mathrm{cm}^{-1}\right)$ v 2954, 2927, 2856, 1769, 1748, 1254, 1112, 838, 779; ${ }^{1} \mathrm{H}$ NMR $(400 \mathrm{MHz}$, $\left.\mathrm{CDCl}_{3}\right) \delta 5.56(\mathrm{dt}, J=14.7,7.1 \mathrm{~Hz}, 1 \mathrm{H}), 5.37(\mathrm{dt}, J=15.1,7.6$ $\mathrm{Hz}, 1 \mathrm{H}$ ), 4.45 (ddt, $J=7.1,4.8,4.0 \mathrm{~Hz}, 1 \mathrm{H}), 3.86$ (dd, $J=10.6$, $4.8 \mathrm{~Hz}, 1 \mathrm{H}), 3.79(\mathrm{dd}, J=10.6,4.0 \mathrm{~Hz}, 1 \mathrm{H}), 3.74(\mathrm{~s}, 0.4 \mathrm{H}), 3.73$ (s, 2.6H), 2.72-2.49 (m, 4H), $2.03(\mathrm{dt}, J=15.6 \mathrm{~Hz}, 7.1 \mathrm{~Hz}, 0.2 \mathrm{H})$, 1.97 (dt, $J=13.6,6.7 \mathrm{~Hz}, 0.8 \mathrm{H}), 1.31$ (br s, 2H), 1.29 (br s, 2H) 1.25 (br s, 8H), 0.88 (br s, 12H), 0.06 (s, 6H); ${ }^{13} \mathrm{C}$ NMR $(100$ $\left.\mathrm{MHz}, \mathrm{CDCl}_{3}\right) \delta$ 209.1, 168.4, 136.8, 135.3, 121.7, 120.9, 86.7, $86.5,76.1,76.0,65.4,52.7,38.9,38.6,32.6,31.9,29.50,29.46$, 29.4, 29.3, 29.1, 27.4, 25,8, 22.6, 18.3, 14.1, -5.5.; HRMS (EI) $\mathrm{m} / z$ calcd for $\mathrm{C}_{24} \mathrm{H}_{44} \mathrm{O}_{5} \mathrm{Si}(\mathrm{M})^{+} 440.2958$, found 440.2954 .

4.2.9. (2R,5S)-Methyl 5-(tert-butyldimethylsilyloxy)methyl-3-(4toluenesulfonyl)hydrazinylidene-2-(2-undecen-1-

yl)tetrahydrofuran-2-carboxylate (20b)

Tosylhydrazine $(212 \mathrm{mg}, 1.14 \mathrm{mmol})$ was added to a solution of ketone $23(313 \mathrm{mg}, 0.71 \mathrm{mmol})$ in THF $(1.1 \mathrm{~mL})$ at room temperature under $\mathrm{N}_{2}$. After stirring at room temperature for $24 \mathrm{~h}$, solvent was removed in vacuo and the residue was purified by column chromatography (silica gel, $15 \%$ EtOAc in hexane) to provide 20b (424 mg, 98\%) as a colorless oil: $[\alpha]_{\mathrm{D}}{ }^{26}+18.2(c 1.0$, $\mathrm{CHCl}_{3}$ ); IR (neat, $\mathrm{cm}^{-1}$ ) v 3209, 2954, 2926, 2855, 1747, 1599, $1463,1435,1409,1349,1254,1170,1094,838,779 ;{ }^{1} \mathrm{H}$ NMR $\left(400 \mathrm{MHz}, \mathrm{CDCl}_{3}\right) \delta 7.84(\mathrm{~d}, J=8.2 \mathrm{~Hz}, 1.6 \mathrm{H}), 7.82(\mathrm{~d}, J=8.2$ $\mathrm{Hz}, 0.4 \mathrm{H}), 7.47(\mathrm{~s}, 0.2 \mathrm{H}), 7.45(\mathrm{~s}, 0.8 \mathrm{H}), 7.31(\mathrm{~d}, J=8.2 \mathrm{~Hz}, 1.6$ $\mathrm{H}), 7.30(\mathrm{~d}, J=8.2 \mathrm{~Hz}, 0.4 \mathrm{H}), 5.44(\mathrm{~m}, 0.2 \mathrm{H}), 5.28(\mathrm{dt}, J=15.4$, $6.4 \mathrm{~Hz}, 0.8 \mathrm{H}), 5.13(\mathrm{dt}, J=15.4,7.1 \mathrm{~Hz}, 1 \mathrm{H}), 4.27(\mathrm{dt}, J=11.4$, $6.9 \mathrm{~Hz}, 1 \mathrm{H}), 3.76(\mathrm{dd}, J=10.5,4.6 \mathrm{~Hz}, 1 \mathrm{H}), 3.60(\mathrm{~s}, 3 \mathrm{H}), 3.59$ $(\mathrm{m}, 1 \mathrm{H}), 2.77-2.38(\mathrm{~m}, 4 \mathrm{H}), 2.43(\mathrm{~s}, 3 \mathrm{H}), 1.97$ (br d, $J=6.4 \mathrm{~Hz}$, $0.4 \mathrm{H}), 1.84$ (br d, $J=6.4 \mathrm{~Hz}, 1.6 \mathrm{H}), 1.26$ (br s, $12 \mathrm{H}), 0.88$ (t, $J=$ $7.1 \mathrm{~Hz}, 3 \mathrm{H}), 0.83$ (s, 9H), $0.01(\mathrm{~s}, 6 \mathrm{H}) ;{ }^{13} \mathrm{C}$ NMR $(100 \mathrm{MHz}$, $\left.\mathrm{CDCl}_{3}\right) \delta 170.3,160.0,144.2,136.2,135.2,129.5,128.1,122.3$, 86.6, 78.7, 65.0, 52.5, 39.6, 32.7, 31.9, 29.6, 29.5, 29.32, 29.30,
29.2, 25.8, 22.7, 21.6, 18.2, 14.1, -5.5; HRMS (EI) $\mathrm{m} / \mathrm{z}$ calcd for $\mathrm{C}_{31} \mathrm{H}_{52} \mathrm{~N}_{2} \mathrm{O}_{6} \mathrm{SSi}(\mathrm{M})^{+}$608.3315, found 608.3326.

4.2.10. (2R,5S)-Methyl 5-(tert-butyldimethylsilyloxy)methyl-2,5dihydro-2-(2-undecen-1-yl)furan-2-carboxylate (21b)

Sodium cyanoborohydride $(270 \mathrm{mg}, 4.30 \mathrm{mmol})$ was added to a solution of tosylhydrazone $\mathbf{2 0 b}(654 \mathrm{mg}, 1.07 \mathrm{mmol})$ in DMF $(54 \mathrm{~mL})$ at room temperature. After refluxing for $1 \mathrm{~h}$, the reaction mixture was diluted with EtOAc $(150 \mathrm{~mL})$ and washed with water $(50 \mathrm{~mL})$ and brine $(50 \mathrm{~mL})$, and dried over anhydrous $\mathrm{Na}_{2} \mathrm{SO}_{4}$. The filtrate was concentrated in vacuo, and the residue was purified by column chromatography (silica gel, 4\% EtOAc in hexane) to provide $21 \mathrm{~b}(345 \mathrm{mg}, 76 \%)$ as a colorless oil: $[\alpha]_{\mathrm{D}}{ }^{26}-$ 44.0 (c 1.0, $\mathrm{CHCl}_{3}$ ); IR (neat, $\mathrm{cm}^{-1}$ ) v 2954, 2926, 2856, 1759, $1735,1255,1095,837,778 ;{ }^{1} \mathrm{H}$ NMR $\left(400 \mathrm{MHz}, \mathrm{CDCl}_{3}\right) \delta 6.00$ $(\mathrm{d}, J=6.0,1.2 \mathrm{~Hz}, 1 \mathrm{H}), 5.87(\mathrm{dd}, J=6.0,1.6 \mathrm{~Hz}, 1 \mathrm{H}), 5.28(\mathrm{dt}, J$ $=15.6,6.8 \mathrm{~Hz}, 1 \mathrm{H}), 5.34(\mathrm{dt}, J=15.6,7.2 \mathrm{~Hz}, 1 \mathrm{H}), 4.85(\mathrm{~m}, 1 \mathrm{H})$, $3.83(\mathrm{dd}, J=10.0,4.4 \mathrm{~Hz}, 1 \mathrm{H}), 3.72(\mathrm{~s}, 0.1 \mathrm{H}), 3.70(\mathrm{~s}, 2.9 \mathrm{H})$, $3.53(\mathrm{dd}, J=9.6,6.8 \mathrm{~Hz}, 1 \mathrm{H}), 2.65(\mathrm{dd}, J=14.6,6.8 \mathrm{~Hz}, 0.1 \mathrm{H})$, 2.55 (dd, $J=14.0,7.2 \mathrm{~Hz}, 0.9 \mathrm{H}), 2.47$ (dd, $J=14.0,7.2 \mathrm{~Hz}$, $0.8 \mathrm{H}), 2.01(\mathrm{dd}, J=14.0,6.8 \mathrm{~Hz}, 0.2 \mathrm{H}), 1.96(\mathrm{dd}, J=14.0,6.8$ $\mathrm{Hz}, 1.8 \mathrm{H}), 1.25$ (br s, 12H), $0.88(\mathrm{t}, J=7.1 \mathrm{~Hz}, 3 \mathrm{H}), 0.88(\mathrm{~s}, 9 \mathrm{H})$, $0.05(\mathrm{~s}, 6 \mathrm{H}) ;{ }^{13} \mathrm{C}$ NMR $\left(100 \mathrm{MHz}, \mathrm{CDCl}_{3}\right) \delta 172.8,135.2,130.6$, 130.4, 129.32, 129.26, 122.9, 93.6, 87.8, 65.9, 52.1, 41.4, 32.6, 31.9, 29.5, 29.4, 29.32, 29.28, 29.1, 25.9, 22.7, 18.3, 14.1, -5.4; HRMS (EI) $m / z$ calcd for $\mathrm{C}_{24} \mathrm{H}_{44} \mathrm{O}_{4} \mathrm{Si}(\mathrm{M})^{+} 424.3009$, found 424.3044 .

4.2.11. (2R,5S)-Methyl 5-(tert-butyldimethylsilyloxy)methyl-2undecanyltetrahydrofuran-2-carboxylate (24)

$\mathrm{Pd} / \mathrm{C}(20 \mathrm{mg}, 10 \mathrm{wt} \%$ of $21 \mathrm{~b})$ was added to the solution of 21b (204 mg, $0.48 \mathrm{mmol}$ ) in $\mathrm{MeOH}(2.4 \mathrm{~mL}$ ) at room temperature. The reaction mixture was placed in an autoclave and was vigorously stirred under $10 \mathrm{~atm}$ of hydrogen for $2 \mathrm{~h}$. The mixture was filtered through a pad of Celite. The filtrate was concentrated in vacuo and the residue was purified by column chromatography (silica gel, 4\% EtOAc in hexane) to provide $\mathbf{2 4}$ $(193 \mathrm{mg}, 94 \%)$ as a colorless oil: $[\alpha]_{\mathrm{D}}{ }^{24}-9.3\left(c 1.0, \mathrm{CHCl}_{3}\right)$; IR (neat, $\mathrm{cm}^{-1}$ ) $\vee 2926,2855,1735,1463,1254,1128,1090,838$, 778; ${ }^{1} \mathrm{H}$ NMR $\left(400 \mathrm{MHz}, \mathrm{CDCl}_{3}\right) \delta: 4.12(\mathrm{tt}, J=6.4,4.6 \mathrm{~Hz}, 1 \mathrm{H})$, $3.78(\mathrm{dd}, J=10.1,4.6 \mathrm{~Hz}, 1 \mathrm{H}), 3.71$ (s, $3 \mathrm{H}), 3.55$ (dd, $J=10.1$, $6.8 \mathrm{~Hz}, 1 \mathrm{H}), 2.35(\mathrm{dt}, J=6.8,4.1 \mathrm{~Hz}, 1 \mathrm{H}), 2.01(\mathrm{~m}, 1 \mathrm{H}), 1.88-$ $1.66(\mathrm{~m}, 4 \mathrm{H}), 1.24$ (br s, 12H), 0.88 (br s, $12 \mathrm{H}), 0.05(\mathrm{~s}, 6 \mathrm{H}) ;{ }^{13} \mathrm{C}$ NMR $\left(100 \mathrm{MHz}, \mathrm{CDCl}_{3}\right) \delta 175.3,87.1,81.0,65.8,52.0,38.6$, 35.0, 31.9, 29.8, 29.6, 29.5, 29.4, 29.3, 28.5, 25.9, 24.3, 22.7, $18.3,14.1,-5.4$; HRMS (EI) $m / z$ calcd for $\mathrm{C}_{24} \mathrm{H}_{48} \mathrm{O}_{4} \mathrm{Si}(\mathrm{M})^{+}$ 428.3322, found 428.3362 .

\subsubsection{2. (2R,5S)-5-(tert-butyldimethylsilyloxy)methyl-2-} undecanyltetrahydrofuran-2-methanol (25)

DIBAL-H (1.0 M in hexane, $1.1 \mathrm{~mL} 1.13 \mathrm{mmol})$ was added dropwise to a solution of methyl ester $24(193 \mathrm{mg}, 0.45 \mathrm{mmol})$ in $\mathrm{CH}_{2} \mathrm{Cl}_{2}(4.5 \mathrm{~mL})$ at $-78{ }^{\circ} \mathrm{C}$ under $\mathrm{N}_{2}$. After stirring at $0{ }^{\circ} \mathrm{C}$ for 15 min, the reaction mixture was quenched by the addition of $\mathrm{MeOH}(2.2 \mathrm{~mL})$ and saturated aqueous $\mathrm{NH}_{4} \mathrm{Cl}(1.1 \mathrm{~mL})$ and then allowed to warm to room temperature. The mixture was dried over anhydrous $\mathrm{Na}_{2} \mathrm{SO}_{4}$ and filtered through a pad of Celite. The filtrate was concentrated in vacuo and the residue was purified by column chromatography (silica gel, 6\% EtOAc in hexane) to provide 25 (161 mg, 89\%) as a colorless oil: $[\alpha]_{\mathrm{D}}{ }^{24}-10.9$ (c 1.0, $\mathrm{CHCl}_{3}$ ); IR (neat, $\mathrm{cm}^{-1}$ ) $\vee 3471,2953,2926,2855,1464,1255$, 1090, 837, 777; ${ }^{1} \mathrm{H}$ NMR $\left(\mathrm{CDCl}_{3}\right) \delta: 4.11$ (br t, $\left.J=7.2 \mathrm{~Hz}, 1 \mathrm{H}\right)$, $3.89(\mathrm{dd}, J=10.8,3.2 \mathrm{~Hz}, 1 \mathrm{H}), 3.57(\mathrm{dt}, J=10.8,2.8 \mathrm{~Hz}, 1 \mathrm{H})$, 3.40 (t, $J=10.8 \mathrm{~Hz}, 2 \mathrm{H}), 3.08$ (dd, $J=10.8,2.8 \mathrm{~Hz}, 1 \mathrm{H}), 2.17-$ $2.00(\mathrm{~m}, 2 \mathrm{H}), 1.91-1.75(\mathrm{~m}, 2 \mathrm{H}), 1.47$ (br t, $J=4.0 \mathrm{~Hz}, 2 \mathrm{H}), 1.25$ (br s, 18H), $0.92(\mathrm{~s}, 9 \mathrm{H}), 0.88(\mathrm{t}, J=6.8 \mathrm{~Hz}, 3 \mathrm{H}), 0.09(\mathrm{~s}, 6 \mathrm{H})$; ${ }^{13} \mathrm{C}$ NMR $\left(100 \mathrm{MHz}, \mathrm{CDCl}_{3}\right) \delta 86.5,79.8,69.3,64.8,37.9,32.5$, 
$31.9,30.3,29.64,29.59,29.58,29.3,28.0,25.9,24.0,22.7,18.5$, 14.1, -5.5; HRMS (EI) $\mathrm{m} / z$ calcd for $\mathrm{C}_{23} \mathrm{H}_{48} \mathrm{O}_{3} \mathrm{Si}(\mathrm{M})^{+} 400.3373$, found 400.3384 .

4.2.13. (2R,5S)-5-(tert-Butyldimethylsilyloxy)methyl-2-
(methoxymethyloxy)methyl-2-undecanyltetrahydrofuran (26)

${ }^{i} \operatorname{Pr}_{2} \mathrm{NEt}(0.2 \mathrm{~mL}, 2.60 \mathrm{mmol})$ and MOMCl $(0.75 \mathrm{~mL}, 4.33$ mmol) was added to the solution of $25(347 \mathrm{mg}, 0.87 \mathrm{mmol})$ in $\mathrm{CH}_{2} \mathrm{Cl}_{2}(4.3 \mathrm{~mL})$ at $0{ }^{\circ} \mathrm{C}$ under $\mathrm{N}_{2}$. After stirring at $0{ }^{\circ} \mathrm{C}$ for 30 $\mathrm{min}$, the reaction mixture was allowed to warm to room temperature and was stirred for $5.5 \mathrm{~h}$. The mixture was quenched by the addition of saturated aqueous $\mathrm{NaHCO}_{3}$ and the mixture was extracted with EtOAc $(3 \times 10 \mathrm{~mL})$. Combined organic layers were washed with water $(10 \mathrm{~mL})$ and brine $(10 \mathrm{~mL})$ and dried over anhydrous $\mathrm{MgSO}_{4}$. The filtrate was concentrated in vacuo, and the residue was purified by column chromatography (silica gel, 6\% EtOAc in hexane) to provide $26(372 \mathrm{mg}, 97 \%)$ as a colorless oil: $[\alpha]_{\mathrm{D}}{ }^{24}-6.5\left(c 1.0, \mathrm{CHCl}_{3}\right)$; IR (neat, $\left.\mathrm{cm}^{-1}\right) \vee 2926$, $2855,1463,1254,1149,1112,1053,837,776 ;{ }^{1} \mathrm{H}$ NMR $(400$ $\left.\mathrm{MHz} \mathrm{CDCl}_{3}\right) \delta 4.63(\mathrm{~s}, 2 \mathrm{H}), 4.03(\mathrm{dt}, J=10.4,6.2 \mathrm{~Hz}, 1 \mathrm{H}), 3.65$ $(\mathrm{dd}, J=10.4,4.2 \mathrm{~Hz}, 1 \mathrm{H}), 3.53(\mathrm{dd}, J=10.4,6.0 \mathrm{~Hz}, 1 \mathrm{H}), 3.41$ $(\mathrm{d}, J=10.0 \mathrm{~Hz}, 1 \mathrm{H}), 3.37(\mathrm{~d}, J=10.0 \mathrm{~Hz}, 1 \mathrm{H}), 3.36(\mathrm{~s}, 3 \mathrm{H})$, $1.99-1.70(\mathrm{~m}, 4 \mathrm{H}), 1.55$ (dd, $J=9.4,5.6 \mathrm{~Hz}, 2 \mathrm{H}), 1.26$ (br s, $18 \mathrm{H}), 0.89$ (br s, 12H), $0.05(\mathrm{~s}, 6 \mathrm{H}) ;{ }^{13} \mathrm{C}$ NMR $(100 \mathrm{MHz}$, $\left.\mathrm{CDCl}_{3}\right) \delta 96.8,84.8,79.9,72.6,65.9,55.2,37.1,32.5,31.9,30.3$, 29.7, 29.6, 29.3, 28.4, 25.9, 24.1, 22.7, 18.4, 14.1; HRMS (EI) $m / z$ calcd for $\mathrm{C}_{25} \mathrm{H}_{52} \mathrm{O}_{4} \mathrm{Si}(\mathrm{M})^{+} 444.3635$, found 444.3658 .

\subsubsection{4. (2R,5S)-2-(Methoxymethyloxy)methyl-2-} undecanyltetrahydrofuran-5-methanol (27)

TBAF (1.0 M in THF, $0.21 \mathrm{~mL}, 0.21 \mathrm{mmol})$ was added to a solution of $26(85 \mathrm{mg}, 0.19 \mathrm{mmol})$ in THF $(1.9 \mathrm{~mL})$ at $0{ }^{\circ} \mathrm{C}$ under $\mathrm{N}_{2}$ and then the resulting mixture was stirred at room temperature. After stirring for $2.5 \mathrm{~h}$, the reaction mixture was diluted with EtOAc $(20 \mathrm{~mL})$ and washed with water $(10 \mathrm{~mL})$ and brine $(10 \mathrm{~mL})$, and dried over anhydrous $\mathrm{Na}_{2} \mathrm{SO}_{4}$. The filtrate was concentrated in vacuo, and the residue was purified by column chromatography (silica gel, $25 \%$ EtOAc in hexane) to provide $27(63 \mathrm{mg}, 100 \%)$ as a colorless oil: $[\alpha]_{\mathrm{D}}{ }^{24}+0.66(c 1.0$, $\mathrm{CHCl}_{3}$ ); IR (neat, $\mathrm{cm}^{-1}$ ) v 3447, 2925, 2854, 1465, 1150, 1112, 1048; ${ }^{1} \mathrm{H}$ NMR $\left(400 \mathrm{MHz}, \mathrm{CDCl}_{3}\right) \delta 4.67(\mathrm{~d}, J=8.4 \mathrm{~Hz}, 1 \mathrm{H})$, $4.66(\mathrm{~d}, J=8.4 \mathrm{~Hz}, 1 \mathrm{H}), 4.17$ (ddd, $J=9.6,6.8,3.2 \mathrm{~Hz}, 1 \mathrm{H})$, $3.79(\mathrm{dt}, J=11.6,3.2 \mathrm{~Hz}, 1 \mathrm{H}), 3.54(\mathrm{~d}, J=10.0 \mathrm{~Hz}, 1 \mathrm{H}), 3.45(\mathrm{~m}$, $1 \mathrm{H}), 3.45(\mathrm{~d}, J=10.0 \mathrm{~Hz}, 1 \mathrm{H}), 3.38(\mathrm{~s}, 3 \mathrm{H}), 2.94(\mathrm{dd}, J=8.6,3.2$ $\mathrm{Hz}, 1 \mathrm{H}), 2.06-1.93(\mathrm{~m}, 3 \mathrm{H}), 1.76(\mathrm{~m}, 1 \mathrm{H}), 1.56-1.49(\mathrm{~m}, 2 \mathrm{H})$, 1.28 (br s, 4H), 1.26 (br s, $14 \mathrm{H}), 0.88(\mathrm{t}, J=7.2 \mathrm{~Hz}, 3 \mathrm{H}) ;{ }^{13} \mathrm{C}$ NMR $\left(100 \mathrm{MHz}, \mathrm{CDCl}_{3}\right) \delta 96.6,85.2,80.1,72.8,65.1,55.4$, $37.9,32.6,31.9,30.2,29.64,29.60,29.58,29.5,29.33,29.31$, 27.6, 24.0, 22.7, 14.1; HRMS (EI) $m / z$ calcd for $\mathrm{C}_{19} \mathrm{H}_{38} \mathrm{O}_{4}(\mathrm{M})^{+}$ 330.2770 , found 330.2779 .

\subsubsection{5.}

(2R,5S)-2-(Methoxymethyloxy)methyl-2undecanyltetrahydrofuran-5-carboaldehyde (28)

A solution of DMSO $(0.044 \mathrm{~mL}, 0.56 \mathrm{mmol})$ in $\mathrm{CH}_{2} \mathrm{Cl}_{2}(0.24$ $\mathrm{mL})$ was dropped to a solution of oxalyl chloride $(0.032 \mathrm{~mL}, 0.38$ mmol) in $\mathrm{CH}_{2} \mathrm{Cl}_{2}(0.4 \mathrm{~mL})$ at $-78{ }^{\circ} \mathrm{C}$ under $\mathrm{N}_{2}$ over $15 \mathrm{~min}$. A solution of alcohol $27(62 \mathrm{mg}, 0.19 \mathrm{mmol})$ in $\mathrm{CH}_{2} \mathrm{Cl}_{2}(0.3 \mathrm{~mL})$ was then dropped to the reaction mixture over $15 \mathrm{~min}$. After stirring for $30 \mathrm{~min}$ at $-78{ }^{\circ} \mathrm{C}$, triethylamine $(0.13 \mathrm{~mL}, 0.94$ mmol) was added to the reaction mixture and the mixture was allowed to warm to room temperature over $80 \mathrm{~min}$. After stirred at room temperature for $40 \mathrm{~min}$, the reaction mixture was quenched by the addition of saturated aqueous $\mathrm{NH}_{4} \mathrm{Cl}(1 \mathrm{~mL})$. The mixture was extracted with $\mathrm{CH}_{2} \mathrm{Cl}_{2}(3 \times 10 \mathrm{~mL})$ and combined organic layers were dried over anhydrous $\mathrm{Na}_{2} \mathrm{SO}_{4}$. The filtrate was concentrated in vacuo, and the residue was purified by column chromatography (silica gel, $20 \%$ EtOAc in hexane to
$50 \%$ EtOAc in hexane) to provide $\mathbf{2 8}(51 \mathrm{mg}, 82 \%)$ as a colorless oil: $[\alpha]_{\mathrm{D}}{ }^{25}-22.2\left(c 1.0, \mathrm{CHCl}_{3}\right)$; IR (neat, $\left.\mathrm{cm}^{-1}\right) \vee$ IR (neat, $\mathrm{cm}^{-}$ $\left.{ }^{1}\right)$ v 3447, 2925, 2854, 1465, 1150, 1112, 1048; ${ }^{1} \mathrm{H}$ NMR $(400$ $\left.\mathrm{MHz}, \mathrm{CDCl}_{3}\right) \delta 9.68(\mathrm{~d}, J=1.6 \mathrm{~Hz}, 1 \mathrm{H}), 4.64(\mathrm{~s}, 2 \mathrm{H}), 4.30(\mathrm{t}, J=$ $10.8 \mathrm{~Hz}, 1 \mathrm{H}), 3.51(\mathrm{~d}, J=10.0 \mathrm{~Hz}, 1 \mathrm{H}), 3.46(\mathrm{~d}, J=10.0 \mathrm{~Hz}$, $1 \mathrm{H}), 3.36(\mathrm{~s}, 3 \mathrm{H}), 2.18-2.10(\mathrm{~m}, 2 \mathrm{H}), 1.93(\mathrm{dt}, J=12.0,6.8 \mathrm{~Hz}$, $1 \mathrm{H}), 1.79$ (dt, $J=12.0,8.0 \mathrm{~Hz}, 1 \mathrm{H}), 1.60$ (br s, 2H), 1.26 (br s, $18 \mathrm{H}), 0.88(\mathrm{t}, J=6.6 \mathrm{~Hz}, 3 \mathrm{H}) ;{ }^{13} \mathrm{C}$ NMR $\left(100 \mathrm{MHz}, \mathrm{CDCl}_{3}\right) \delta$ 203.8, 96.7, 87.1, 83.7, 72.7, 55.4, 37.3, 32.2, 31.9, 30.2, 29.63, 29.60, 29.57, 29.3, 28.0, 24.0, 22.7, 14.1; HRMS (EI) $\mathrm{m} / \mathrm{z}$ calcd for $\mathrm{C}_{19} \mathrm{H}_{38} \mathrm{O}_{4}(\mathrm{M})^{+} 328.2614$, found 328.2616.

\subsubsection{6. (S)-5-(Methoxymethyloxy)methyl-5- undecanyltetrahydropyran-1-one (30)}

1,3-Bis(2,6-diisopropylphenyl)imidazolinium chloride (29) (20 mg, $0.05 \mathrm{mmol}, 30 \mathrm{~mol} \%)$ and DBU $(0.005 \mathrm{~mL}, 0.031 \mathrm{mmol}$, $20 \mathrm{~mol} \%)$ was added to the solution of aldehyde 28 (51 mg, 0.16 mmol) in $\mathrm{CHCl}_{3}(3.1 \mathrm{~mL})$ at room temperature under $\mathrm{N}_{2}$. After refluxing for $24 \mathrm{~h}$, the reaction mixture was quenched by the addition of saturated aqueous $\mathrm{NH}_{4} \mathrm{Cl}(3 \mathrm{~mL})$ at room temperature. The mixture was extracted with $\mathrm{CH}_{2} \mathrm{Cl}_{2}(3 \times 10 \mathrm{~mL})$ and combined organic layers were dried over anhydrous $\mathrm{Na}_{2} \mathrm{SO}_{4}$. The filtrate was concentrated in vacuo, and the residue was purified by column chromatography (silica gel, $20 \%$ EtOAc in hexane) to provide $30(36 \mathrm{mg}, 71 \%)$ as a colorless oil: $[\alpha]_{\mathrm{D}}{ }^{27}-2.8(c 1.0$, $\mathrm{CHCl}_{3}$ ); IR (neat, $\left.\mathrm{cm}^{-1}\right) \vee 2925,2854,1738,1465,1330,1248$, 1150, 1114, 1050, 921; ${ }^{1} \mathrm{H}$ NMR (400 MHz, $\left.\mathrm{CDCl}_{3}\right) \delta 4.63(\mathrm{~s}$, $2 \mathrm{H}), 3.56(\mathrm{~d}, J=10.0 \mathrm{~Hz}, 1 \mathrm{H}), 3.51(\mathrm{~d}, J=10.0 \mathrm{~Hz}, 1 \mathrm{H}), 3.36(\mathrm{~s}$, $3 \mathrm{H}), 2.50$ (dt, $J=18.0,6.8 \mathrm{~Hz}, 1 \mathrm{H}), 2.44$ (dt, $J=18.0,6.8 \mathrm{~Hz}$, $1 \mathrm{H}), 1.98(\mathrm{dt}, J=12.4,4.4 \mathrm{~Hz}, 1 \mathrm{H}), 1.92(\mathrm{dt}, J=10.4,3.6 \mathrm{~Hz}$, $1 \mathrm{H}), 1.85-1.60(\mathrm{~m}, 4 \mathrm{H}), 1.26($ br s, $18 \mathrm{H}), 0.88$ (t, $J=7.2 \mathrm{~Hz}$, $3 \mathrm{H}) ;{ }^{13} \mathrm{C}$ NMR $\left(100 \mathrm{MHz}, \mathrm{CDCl}_{3}\right) \delta 171.5,96.7,84.6,71.8,55.4$, 38.0, 31.9, 29.9, 29.8, 29.6, 29.59, 29.58, 29.52, 29.46, 29.3, 27.7, 23.0, 22.7, 16.8, 14.1; HRMS (EI) $\mathrm{m} / z$ calcd for $\mathrm{C}_{19} \mathrm{H}_{38} \mathrm{O}_{4}(\mathrm{M})^{+}$ 328.2614 , found 328.2648 .

\subsubsection{7. (+)-Tanikolide (1)}

Aqueous $\mathrm{HCl}(4 \mathrm{M}, 1.8 \mathrm{~mL})$ was added dropwise to the solution of MOM ether $\mathbf{3 0}(120 \mathrm{mg}, 0.37 \mathrm{mmol})$ in THF $(1.8 \mathrm{~mL})$ at room temperature. After stirring the reaction mixture at $50{ }^{\circ} \mathrm{C}$ for $6 \mathrm{~h}$, the reaction mixture was saturated with $\mathrm{NaCl}$. The mixture was extracted with $\mathrm{CHCl}_{3}(3 \times 20 \mathrm{~mL})$ and combined organic layers were dried over anhydrous $\mathrm{Na}_{2} \mathrm{SO}_{4}$. The filtrate was concentrated in vacuo, and the residue was purified by column chromatography (silica gel, $40 \%$ EtOAc in hexane) to provide 1 (59 mg, 56\%) as a white solid: $\mathrm{mp} 39-40{ }^{\circ} \mathrm{C}$ (lit. ${ }^{6 \mathrm{~h}}$ $\left.39-41{ }^{\circ} \mathrm{C}\right) ;[\alpha]_{\mathrm{D}}{ }^{24}+2.2\left(c 0.65, \mathrm{CHCl}_{3}\right)\left(\right.$ lit. $^{1}[\alpha]_{\mathrm{D}}{ }^{25}+2.3(c 0.65$, $\left.\mathrm{CHCl}_{3}\right)$ ); IR (neat, $\mathrm{cm}^{-1}$ ) v 3395, 2923, 2851, 1701, 1468, 1265, $1051 ;{ }^{1} \mathrm{H}$ NMR $\left(400 \mathrm{MHz}, \mathrm{CDCl}_{3}\right) \delta 3.67(\mathrm{~d}, J=12.0 \mathrm{~Hz}, 1 \mathrm{H})$, $3.54(\mathrm{~d}, J=12.0 \mathrm{~Hz}, 1 \mathrm{H}), 2.94(\mathrm{br} \mathrm{s}, 1 \mathrm{H}), 2.47(\mathrm{t}, J=6.6 \mathrm{~Hz}, 1 \mathrm{H})$, $1.97-1.80(\mathrm{~m}, 3 \mathrm{H}), 1.75-1.61(\mathrm{~m}, 3 \mathrm{H}), 1.26(\mathrm{br} \mathrm{s}, 18 \mathrm{H}), 0.88(\mathrm{t}, J$ $=6.8 \mathrm{~Hz}, 3 \mathrm{H}) ;{ }^{13} \mathrm{C} \mathrm{NMR}\left(100 \mathrm{MHz}, \mathrm{CDCl}_{3}\right) \delta 172.0,86.6,67.3$, 36.7, 31.8, 29.9, 29.7, 29.53, 29.51, 29.47, 29.3, 29.2. 26.6, 23.3, $22.6,16.6,14.0$.

\subsection{Synthesis of (4S)- and (4R)-hydroxytanikolides (2)}

4.3.1. $(2 R, 3 S, 5 S)$ - and $(2 R, 3 R, 5 S)$-2-allyl-5-(tertbutyldimethylsilyloxy)methyl-3-hydroxytetrahydrofuran-2methanol $\{(3 S)-8 \boldsymbol{a}\}$ and $\{(3 R)-8 \boldsymbol{a}\}$

$\mathrm{LiAlH}_{4}(229 \mathrm{mg}, 6.02 \mathrm{mmol})$ was added to a solution of 3oxotetrahydrofuran 6a $(989 \mathrm{mg}, 3.01 \mathrm{mmol})$ in THF $(15 \mathrm{~mL})$ at $0{ }^{\circ} \mathrm{C}$ under $\mathrm{N}_{2}$. After stirring at $0{ }^{\circ} \mathrm{C}$ for $4.5 \mathrm{~h}$, the reaction mixture was diluted by excess amount of $\mathrm{Et}_{2} \mathrm{O}$ and quenched by addition of $\mathrm{H}_{2} \mathrm{O}(1.1 \mathrm{~mL})$ and $15 \%$ aqueous $\mathrm{NaOH}(0.3 \mathrm{~mL})$ and stirred at room temperature for $1 \mathrm{~h}$. The mixture was filtered through a pad of Celite. The filtrate was concentrated in vacuo 
and the residue was purified by column chromatography (silica gel, 25\% EtOAc in hexane) to provide (3S)-8a (471 mg, 52\%) as a colorless oil and (3R)-8a (337 mg, 37\%) as a white solid. (3S)8a: $[\alpha]_{\mathrm{D}}{ }^{24}+17.9\left(c 1.0, \mathrm{CHCl}_{3}\right)$; IR (neat, $\left.\mathrm{cm}^{-1}\right) \vee 3419,3076$, 2954, 2929, 2858, 1640, 1471, 1256, 1091, 837, 778; ${ }^{1}$ H NMR $\left(400 \mathrm{MHz}, \mathrm{CDCl}_{3}\right) \delta 5.83(\mathrm{ddt}, J=17.6,9.6,7.2 \mathrm{~Hz}, 1 \mathrm{H}), 5.14$ (dd, $J=7.2,1.2 \mathrm{~Hz}, 1 \mathrm{H}), 5.10(\mathrm{br} \mathrm{s}, 1 \mathrm{H}), 4.20-4.13(\mathrm{~m}, 2 \mathrm{H}), 3.92$ $(\mathrm{dd}, J=11.0,2.4 \mathrm{~Hz}, 1 \mathrm{H}), 3.70(\mathrm{~d}, J=11.6 \mathrm{~Hz}, 1 \mathrm{H}), 3.70(\mathrm{~d}, J=$ $6.0 \mathrm{~Hz}, 1 \mathrm{H}), 3.62(\mathrm{~d}, J=11.6 \mathrm{~Hz}, 1 \mathrm{H}), 3.54(\mathrm{dd}, J=11.0,2.4 \mathrm{~Hz}$, $1 \mathrm{H}), 3.08(\mathrm{dd}, J=7.6,6.0 \mathrm{~Hz}, 1 \mathrm{H}), 2.43(\mathrm{dt}, J=13.2,7.6 \mathrm{~Hz}$, 1H), 2.24 (ddt, $J=13.8,7.6,1.2 \mathrm{~Hz}, 2 \mathrm{H}), 2.03$ (ddd, $J=13.2,6.0$, $5.6 \mathrm{~Hz}, 1 \mathrm{H}), 0.93(\mathrm{~s}, 9 \mathrm{H}), 0.12(\mathrm{~s}, 6 \mathrm{H}) ;{ }^{13} \mathrm{C}$ NMR $(100 \mathrm{MHz}$, $\left.\mathrm{CDCl}_{3}\right) \delta 133.1,118.4,86.7,77.0,76.4,65.9,65.0,40.0,36.7$, $25.9,18.5,-5.51$; HRMS (EI) $\mathrm{m} / \mathrm{z}$ calcd for $\mathrm{C}_{15} \mathrm{H}_{30} \mathrm{O}_{4} \mathrm{Si}(\mathrm{M})^{+}$ 302.1913, found 302.1915. (3R)-8a: $\mathrm{mp} 43-44{ }^{\circ} \mathrm{C},[\alpha]_{\mathrm{D}}{ }^{25}-8.0(c$ $\left.1.0, \mathrm{CHCl}_{3}\right)$; IR $\left(\mathrm{KBr}, \mathrm{cm}^{-1}\right) \vee 3323,3075,2952,2931,2868$, $1641,1464,1254,1097,1033,996,836,777 ;{ }^{1} \mathrm{H}$ NMR (400 $\left.\mathrm{MHz}, \mathrm{CDCl}_{3}\right) \delta 5.90(\mathrm{ddt}, J=17.4,10.0,7.2 \mathrm{~Hz}, 1 \mathrm{H}), 5.18$ (ddd, $J=17.4,3.6,1.2 \mathrm{~Hz}, 1 \mathrm{H}), 5.13$ (ddd, $J=10.0,1.2,0.8 \mathrm{~Hz}, 1 \mathrm{H})$, $4.40(\mathrm{dd}, J=6.8,3.6 \mathrm{~Hz}, 1 \mathrm{H}), 4.35(\mathrm{ddt}, J=9.4,6.8,2.4 \mathrm{~Hz}, 1 \mathrm{H})$ $3.93(\mathrm{dd}, J=11.2,6.8 \mathrm{~Hz}, 1 \mathrm{H}), 3.59(\mathrm{dd}, J=11.6,3.6 \mathrm{~Hz}, 1 \mathrm{H})$, $3.57(\mathrm{dd}, J=11.2,1.6 \mathrm{~Hz}, 1 \mathrm{H}), 3.48(\mathrm{dd}, J=11.6,9.2 \mathrm{~Hz}, 1 \mathrm{H})$, 2.99 (dd, $J=9.4,3.6 \mathrm{~Hz}, 1 \mathrm{H}), 2.45$ (br d, $J=7.2 \mathrm{~Hz}, 2 \mathrm{H}), 2.44$ (m, $1 \mathrm{H}), 1.88$ (ddd, $J=13.4,7.2,3.2 \mathrm{~Hz}, 1 \mathrm{H}), 1.82$ (br d, $J=3.6$ $\mathrm{Hz}, 1 \mathrm{H}), 0.91(\mathrm{~s}, 9 \mathrm{H}), 0.09$ (s, 6H); ${ }^{13} \mathrm{C}$ NMR $\left(100 \mathrm{MHz}, \mathrm{CDCl}_{3}\right)$ $\delta 134.5,118.1,87.6,77.7,75.3,67.0,64.5,37.1,36.4,25.9,18.5$, -5.5 ; HRMS (EI) $\mathrm{m} / \mathrm{z}$ calcd for $\mathrm{C}_{15} \mathrm{H}_{30} \mathrm{O}_{4} \mathrm{Si}(\mathrm{M})^{+} 302.1913$, found 302.1872 .

4.3.2. (4aS, 6S, 7aS)-4a-Allyl-6-(tert-butyldimethylsilyloxy)methyl2,2-dimethyl-4H-furo [3,2-d][1,3] dioxine $\{(3 S)-31\}$

$\mathrm{TsOH} \cdot \mathrm{H}_{2} \mathrm{O}(22 \mathrm{mg}, 0.11 \mathrm{mmol})$ was added to a solution of diol (3S)-8a (574 mg, $1.90 \mathrm{mmol}$ ) and 2,2-dimethoxypropane $(2.3 \mathrm{~mL}, 19.0 \mathrm{mmol})$ in benzene $(19 \mathrm{~mL})$ at room temperature. After refluxing for $30 \mathrm{~min}$, the reaction mixture was quenched by saturated $\mathrm{NaHCO}_{3}$ at room temperature. After stirring for $15 \mathrm{~min}$ at room tempertue, the reaction mixture was diluted with EtOAc $(20 \mathrm{~mL})$ and washed with water $(20 \mathrm{~mL})$ and brine $(20 \mathrm{~mL})$, and dried over anhydrous $\mathrm{Na}_{2} \mathrm{SO}_{4}$. The filtrate was concentrated in vacuo, and the residue was purified by column chromatography (silica gel, 5\% EtOAc in hexane) to provide acetonide (3S)-31 $(622 \mathrm{mg}, 96 \%)$ as a colorless oil: $[\alpha]_{\mathrm{D}}{ }^{24}+17.1\left(\mathrm{c} 1.0, \mathrm{CHCl}_{3}\right)$; IR $\left(\right.$ neat, $\left.\mathrm{cm}^{-1}\right) \vee 3077,2986,2954,2929,2857,1641,1472,1373$, 1256, 1227, 1094, 836, 777; ${ }^{1} \mathrm{H}$ NMR $\left(400 \mathrm{MHz}, \mathrm{CDCl}_{3}\right) \delta 5.82$ $(\mathrm{m}, 1 \mathrm{H}), 5.13(\mathrm{~s}, 1 \mathrm{H}), 5.10(\mathrm{~d}, J=8.0 \mathrm{~Hz}, 1 \mathrm{H}), 4.17$ (dddd, $J=$ 11.6, 5.8, 5.2, $1.2 \mathrm{~Hz}, 1 \mathrm{H}), 4.07$ (d, $J=5.6 \mathrm{~Hz}, 1 \mathrm{H}), 3.78$ (dd, $J=$ 9.6, 1.6 Hz, 1H), $3.73(\mathrm{~d}, J=12.0 \mathrm{~Hz}, 1 \mathrm{H}), 3.65(\mathrm{dd}, J=9.6,8.0$ $\mathrm{Hz}, 1 \mathrm{H}), 3.63(\mathrm{~d}, J=12.0 \mathrm{~Hz}, 1 \mathrm{H}), 2.31-2.15(\mathrm{~m}, 3 \mathrm{H}), 1.92(\mathrm{dd}$, $J=13.6,2.8 \mathrm{~Hz}, 1 \mathrm{H}), 1.36(\mathrm{~s}, 3 \mathrm{H}), 1.35$ (s, 3H), $0.90(\mathrm{~s}, 9 \mathrm{H})$, $0.08(\mathrm{~s}, 6 \mathrm{H}) ;{ }^{13} \mathrm{C}$ NMR $\left(100 \mathrm{MHz}, \mathrm{CDCl}_{3}\right) \delta 132.7,118.5,98.3$, 82.1, 79.1, 74.5, 66.5, 64.9, 40.2, 34.5, 26.9, 26.0, 21.2, 18.4, 5.2; HRMS (EI) $\mathrm{m} / \mathrm{z}$ calcd for $\mathrm{C}_{18} \mathrm{H}_{35} \mathrm{O}_{4} \mathrm{Si}(\mathrm{M}+\mathrm{H})^{+}$343.2305, found 343.2294 .

\subsection{3. (4aS, 6S, 7aS)-4a-Allyl-2,2-dimethyl-4H-furo[3,2- d] [1,3]dioxine-6-methanol $\{(3 S)-44\}$}

TBAF $(1.0 \mathrm{M}$ in THF, $1.0 \mathrm{~mL}, 1.00 \mathrm{mmol})$ was added to a solution of silyl ether (3S)-31 (312 mg, $0.91 \mathrm{mmol})$ in THF $(9.0$ $\mathrm{mL}$ ) at $0{ }^{\circ} \mathrm{C}$ under $\mathrm{N}_{2}$ and then the resulting mixture was stirred at room temperature. After stirring for $1.5 \mathrm{~h}$, the reaction mixture was diluted with EtOAc $(30 \mathrm{~mL})$ and washed with water $(15 \mathrm{~mL})$ and brine $(15 \mathrm{~mL})$, and dried over anhydrous $\mathrm{Na}_{2} \mathrm{SO}_{4}$. The filtrate was concentrated in vacuo, and the residue was purified by column chromatography (silica gel, $45 \%$ EtOAc in hexane) to provide alcohol $(3 S)-\mathbf{4 4}(206 \mathrm{mg}, 99 \%)$ as a colorless oil: $[\alpha]_{\mathrm{D}}{ }^{25}$ +53.8 (c 1.0, $\mathrm{CHCl}_{3}$ ); IR (neat, $\mathrm{cm}^{-1}$ ) v 3464, 3076, 2988, 2940,
$1639,1438,1376,1227,1201,1159,1052,916,862 ;{ }^{1} \mathrm{H}$ NMR $\left(400 \mathrm{MHz}, \mathrm{CDCl}_{3}\right) \delta 5.81(\mathrm{~m}, 1 \mathrm{H}), 5.15(\mathrm{~d}, J=7.6 \mathrm{~Hz}, 1 \mathrm{H}), 5.14$ $(\mathrm{dd}, J=18.0,1.6 \mathrm{~Hz}, 1 \mathrm{H}), 4.33(\mathrm{~m}, 1 \mathrm{H}), 4.15(\mathrm{~d}, J=5.6 \mathrm{~Hz}, 1 \mathrm{H})$, $3.79(\mathrm{~d}, J=6.8 \mathrm{~Hz}, 2 \mathrm{H}), 3.78(\mathrm{~m}, 1 \mathrm{H}), 3.65(\mathrm{~m}, 1 \mathrm{H}), 2.69$ (t, $J=$ $5.0 \mathrm{~Hz}, 1 \mathrm{H}), 2.41(\mathrm{dt}, J=14.4,5.6 \mathrm{~Hz}, 1 \mathrm{H}), 2.23(\mathrm{dd}, J=14.4$, $7.2 \mathrm{~Hz}, 1 \mathrm{H}), 2.15(\mathrm{dd}, J=14.4,7.6 \mathrm{~Hz}, 1 \mathrm{H}), 1.91(\mathrm{dd}, J=14.4$, $3.4 \mathrm{~Hz}, 1 \mathrm{H}), 1.40(\mathrm{~s}, 3 \mathrm{H}), 1.39$ (s, 3H); ${ }^{13} \mathrm{C}$ NMR $(100 \mathrm{MHz}$, $\left.\mathrm{CDCl}_{3}\right) \delta 132.1,118.9,98.3,81.1,78.1,73.9,65.2,64.4,39.4$, 34.0, 27.4, 20.4; HRMS (EI) $\mathrm{m} / \mathrm{z}$ calcd for $\mathrm{C}_{12} \mathrm{H}_{20} \mathrm{O}_{4}(\mathrm{M})^{+}$ 228.1362 , found 228.1359 .

\subsection{4. (4aS, 6S,7aS)-4a-Allyl-2,2-dimethyl-4H-furo[3,2- d] [1,3] dioxine-6-carboaldehyde $\{(3 S)-32\}$}

A solution of DMSO $(284 \mathrm{mg}, 3.64 \mathrm{mmol})$ in $\mathrm{CH}_{2} \mathrm{Cl}_{2}(1.3$ $\mathrm{mL}$ ) was dropped to a solution of oxalyl chloride (369 mg, 2.91 mmol) in $\mathrm{CH}_{2} \mathrm{Cl}_{2}(2.0 \mathrm{~mL})$ at $-78{ }^{\circ} \mathrm{C}$ under $\mathrm{N}_{2}$ over $15 \mathrm{~min}$. A solution of alcohol $(3 S)-44(332 \mathrm{mg}, 1.45 \mathrm{mmol})$ in $\mathrm{CH}_{2} \mathrm{Cl}_{2}(1.5$ $\mathrm{mL}$ ) was then dropped to the reaction mixture over $15 \mathrm{~min}$. After stirring for $30 \mathrm{~min}$ at $-78{ }^{\circ} \mathrm{C}$, triethylamine $(1.0 \mathrm{~mL}, 7.27 \mathrm{mmol})$ was added to the reaction mixture and the mixture was allowed to warm to room temperature over $60 \mathrm{~min}$. After stirred at room temperature for $30 \mathrm{~min}$, the reaction mixture was quenched by the addition of saturated aqueous $\mathrm{NH}_{4} \mathrm{Cl}(10 \mathrm{~mL})$. The mixture was extracted with $\mathrm{CH}_{2} \mathrm{Cl}_{2}(3 \times 15 \mathrm{~mL})$ and combined organic layers were dried over anhydrous $\mathrm{Na}_{2} \mathrm{SO}_{4}$. The filtrate was concentrated in vacuo, and the residue was purified by column chromatography (silica gel, $25 \%$ EtOAc in hexane) to provide aldehyde $(3 S)-32(290 \mathrm{mg}, 88 \%)$ as a colorless oil: $[\alpha]_{\mathrm{D}}{ }^{26}+37.6$ $\left(c 1.0, \mathrm{CHCl}_{3}\right)$; IR (neat, $\left.\mathrm{cm}^{-1}\right) \vee 2990,2939,2817,1732,1639$, 1449, 1438, 1375, 1227, 1202, 1159, 1096, 1077, 1058, 1024, 921, 857, 821; ${ }^{1} \mathrm{H}$ NMR $\left(400 \mathrm{MHz}, \mathrm{CDCl}_{3}\right) \delta 9.81(\mathrm{~s}, 1 \mathrm{H}), 5.81$ (ddt, $J=17.6,9.6,7.6 \mathrm{~Hz}, 1 \mathrm{H}), 5.17(\mathrm{~d}, J=9.6 \mathrm{~Hz}, 1 \mathrm{H}), 5.15(\mathrm{~d}$, $J=17.6 \mathrm{~Hz}, 1 \mathrm{H}), 4.38(\mathrm{~d}, J=10.0 \mathrm{~Hz}, 1 \mathrm{H}), 4.16(\mathrm{~d}, J=4.0 \mathrm{~Hz}$, $1 \mathrm{H}), 3.85$ (s, 2H), 2.50 (ddd, $J=13.8,9.8,4.0 \mathrm{~Hz}, 1 \mathrm{H}), 2.28-$ $2.15(\mathrm{~m}, 3 \mathrm{H}), 1.38(\mathrm{~s}, 3 \mathrm{H}), 1.32(\mathrm{~s}, 3 \mathrm{H}) ;{ }^{13} \mathrm{C}$ NMR $(100 \mathrm{MHz}$, $\left.\mathrm{CDCl}_{3}\right) \delta 205.1,131.9,119.2,97.9,82.4,82.2,72.8,64.3,40.8$, 37.3, 27.5, 20.5; HRMS (EI) $\mathrm{m} / \mathrm{z}$ calcd for $\mathrm{C}_{12} \mathrm{H}_{18} \mathrm{O}_{4}(\mathrm{M})^{+}$ 226.1205 , found 226.1211 .

\subsection{5. (4aS,8aS)-4a-Allyl-2,2-dimethyl-4H-pyrano[3,2- d] [1,3] dioxine-6-one $\{(4 S)-33\}$}

Catalyst 29 (30 mg, $0.07 \mathrm{mmol}, 30 \mathrm{~mol} \%)$ and DBU (0.007 $\mathrm{mL}, 0.05 \mathrm{mmol}, 20 \mathrm{~mol} \%$ ) was added to the solution of aldehyde (3S)-32 (53 mg, $0.23 \mathrm{mmol})$ in $\mathrm{CHCl}_{3}(7.0 \mathrm{~mL})$ at room temperature under $\mathrm{N}_{2}$. After refluxing for $10 \mathrm{~h}$, the reaction mixture was quenched by the addition of saturated aqueous $\mathrm{NH}_{4} \mathrm{Cl}(3 \mathrm{~mL})$ at room temperature. The mixture was extracted with $\mathrm{CH}_{2} \mathrm{Cl}_{2}(3 \times 10 \mathrm{~mL})$ and combined organic layers were dried over anhydrous $\mathrm{Na}_{2} \mathrm{SO}_{4}$. The filtrate was concentrated in vacuo, and the residue was purified by column chromatography (silica gel, 30\% EtOAc in hexane) to provide lactone (4S)-33 (40 mg, $75 \%$ ) as a white solid: $\mathrm{mp} 85-86{ }^{\circ} \mathrm{C}$; $[\alpha]_{\mathrm{D}}{ }^{24}+100.3$ (c 1.0, $\left.\mathrm{CHCl}_{3}\right)$; IR $\left(\mathrm{KBr}, \mathrm{cm}^{-1}\right) \vee$ 3004, 2986, 2971, 2954, 2909, 1731, $1643,1456,1388,1378,1350,1314,1263,1209,1175,1156$, $1119,1086,1037,1007,986,941,863,732 ;{ }^{1} \mathrm{H}$ NMR $(400 \mathrm{MHz}$, $\left.\mathrm{CDCl}_{3}\right) \delta 5.81$ (dddd, 16.9, 9.6, 7.3, 6.6 Hz, 1H), $5.21(\mathrm{~d}, J=9.6$ $\mathrm{Hz}, 1 \mathrm{H}), 5.18(\mathrm{dd}, J=16.9,1.4 \mathrm{~Hz}, 1 \mathrm{H}), 4.03(\mathrm{t}, J=3.2 \mathrm{~Hz}, 1 \mathrm{H})$, $3.81(\mathrm{~d}, J=12.6 \mathrm{~Hz}, 1 \mathrm{H}), 3.70(\mathrm{~d}, J=12.6 \mathrm{~Hz}, 1 \mathrm{H}), 2.76$ (ddd, $J$ $=18.2,12.8,7.3 \mathrm{~Hz}, 1 \mathrm{H}), 2.49-2.43(\mathrm{~m}, 2 \mathrm{H}), 2.31(\mathrm{dd}, J=14.4$, $8.0 \mathrm{~Hz}, 1 \mathrm{H}), 2.10(\mathrm{~m} \mathrm{1H}), 1.94$ (dddd, $J=14.4,7.4,3.2,1.8 \mathrm{~Hz}$, 1H), $1.44(\mathrm{~s}, 3 \mathrm{H}), 1.39$ (s, 3H); ${ }^{13} \mathrm{C}$ NMR $\left(100 \mathrm{MHz}, \mathrm{CDCl}_{3}\right) \delta$ 170.4, 130.4, 120.3, 98.7, 79.7, 65.5, 64.9, 40.9, 27.6, 24.3, 22.2, 20.0; HRMS (EI) $\mathrm{m} / \mathrm{z}$ calcd for $\mathrm{C}_{12} \mathrm{H}_{18} \mathrm{O}_{4}(\mathrm{M})^{+} 226.1205$, found 226.1243

4.3.6. (4aS,8aS)-2,2-dimethyl-4a-(2-undecen-1-yl)-4Hpyrano $[3,2-d][1,3]$ dioxine-6-one $\{(4 S)-45\}$ 
Grubbs 2nd catalyst $(3.3 \mathrm{mg}, 0.004 \mathrm{mmol})$ was added to the solution of (4S)-33 $(87 \mathrm{mg}, 0.38 \mathrm{mmol})$ and 1-decene $(0.36 \mathrm{~mL}$, $1.92 \mathrm{mmol})$ in $\mathrm{CH}_{2} \mathrm{Cl}_{2}(3.8 \mathrm{~mL})$ at room temperature under $\mathrm{N}_{2}$. After refluxing for $3 \mathrm{~h}, 1$-decene $(0.36 \mathrm{~mL}, 1.92 \mathrm{mmol})$ and Grubbs 2nd catalyst $(3.3 \mathrm{mg}, 0.004 \mathrm{mmol})$ were added to the reaction mixture and the mixture was refluxed for $3 \mathrm{~h}$. Solvent was removed in vacuo, and the residue was purified by column chromatography (silica gel, 20\% EtOAc in hexane) to provide $(4 S)-45(119 \mathrm{mg}, 92 \%)$ as a colorless oil: $[\alpha]_{\mathrm{D}}{ }^{24}+59.0(c$ 1.0, $\mathrm{CHCl}_{3}$ ); IR (neat, $\mathrm{cm}^{-1}$ ) v 2991, 2925, 2854, 1733, 1456, 1377, $1250,1205,1162,1090,1059,1003,989,938,864 ;{ }^{1} \mathrm{H}$ NMR $\left(400 \mathrm{MHz}, \mathrm{CDCl}_{3}\right) \delta 5.56(\mathrm{dt}, J=15.1,6.9 \mathrm{~Hz}, 1 \mathrm{H}), 5.38(\mathrm{ddd}, J$ $=15.1,7.8,6.9 \mathrm{~Hz}, 1 \mathrm{H}), 4.02(\mathrm{t}, J=3.2 \mathrm{~Hz}, 1 \mathrm{H}), 3.80(\mathrm{~d}, J=$ $12.8 \mathrm{~Hz}, 1 \mathrm{H}), 3.68(\mathrm{~d}, J=12.8 \mathrm{~Hz}, 1 \mathrm{H}), 2.75(\mathrm{ddd}, J=18.1,12.5$, $7.3 \mathrm{~Hz}, 1 \mathrm{H}), 2.45$ (ddd, $J=18.1,6.4,1.8 \mathrm{~Hz}, 1 \mathrm{H}), 2.39$ (dd, $J=$ $14.4,6.4 \mathrm{~Hz}, 1 \mathrm{H}), 2.24$ (dd, $J=14.4,7.8 \mathrm{~Hz}, 1 \mathrm{H}), 2.15-2.11(\mathrm{~m}$, 1H), 2.02 (dd, $J=14.0,6.9 \mathrm{~Hz}, 2 \mathrm{H}$ ), 1.92 (dddd, $J=14.5,7.3$, $3.2,1.8 \mathrm{~Hz}, 1 \mathrm{H}), 1.43(\mathrm{~s}, 3 \mathrm{H}), 1.39(\mathrm{~s}, 3 \mathrm{H}), 1.35-1.30(\mathrm{~m}, 3 \mathrm{H})$, 1.26 (br s, 9H), 0.88 (t, $J=7.3 \mathrm{~Hz}, 3 \mathrm{H}) ;{ }^{13} \mathrm{C} \mathrm{NMR}(100 \mathrm{MHz}$, $\left.\mathrm{CDCl}_{3}\right) \delta 170.5,136.7,121.5,98.6,80.0,65.6,64.8,39.7,32.5$, $31.8,29.4,29.2,29.2,29.1,27.7,24.4,22.6,22.2,19.9,14.1$; HRMS (EI) $\mathrm{m} / \mathrm{z}$ calcd for $\mathrm{C}_{20} \mathrm{H}_{34} \mathrm{O}_{4}(\mathrm{M})^{+}$338.2457, found 338.2420 .

4.3.7. (4aS,8aS)-2,2-dimethyl-4a-undecyl-4H-pyrano[3,2d] [1,3] dioxine-6-one $\{(4 S)-34\}$

$\mathrm{Pd} / \mathrm{C}\{23 \mathrm{mg}, 10 \mathrm{wt} \%$ of $(4 S)-45\}$ was added to the solution of $(4 S)-45(231 \mathrm{mg}, 0.68 \mathrm{mmol})$ in $\mathrm{MeOH}(3.4 \mathrm{~mL})$ at room temperature. The reaction mixture was vigorously stirred under $\mathrm{H}_{2}$ atmosphere for $1.5 \mathrm{~h}$. The mixture was filtered through a pad of Celite. The filtrate was concentrated in vacuo and the residue was purified by column chromatography (silica gel, 25\% EtOAc in hexane) to provide (4S)-34 (219 $\mathrm{mg}, 94 \%)$ as a white solid: $\mathrm{mp}$ $62-63{ }^{\circ} \mathrm{C} ;[\alpha]_{\mathrm{D}}{ }^{24}+55.1\left(\mathrm{c} 1.0, \mathrm{CHCl}_{3}\right)$; IR $\left(\mathrm{KBr}, \mathrm{cm}^{-1}\right)$ v 3004, 2945, 2914, 2852, 1725, 1473, 1380, 1262, 1212, 1149, 1092, $1042,989,939,863 ;{ }^{1} \mathrm{H}$ NMR $\left(400 \mathrm{MHz}, \mathrm{CDCl}_{3}\right) \delta 3.99(\mathrm{t}, J=$ $2.8 \mathrm{~Hz}, 1 \mathrm{H}), 3.81(\mathrm{~d}, J=12.8 \mathrm{~Hz}, 1 \mathrm{H}), 3.76(\mathrm{~d}, J=12.8 \mathrm{~Hz}, 1 \mathrm{H})$, 2.76 (ddd, $J=18.4,12.4,7.2 \mathrm{~Hz}, 1 \mathrm{H}$ ), 2.45 (ddd, $J=18.4,5.4$, $1.6 \mathrm{~Hz}, 1 \mathrm{H}), 2.09$ (m, 1H), 1.93 (dddd, $J=14.6,7.2,3.6,1.6 \mathrm{~Hz}$, $1 \mathrm{H}), 1.66-1.49(\mathrm{~m}, 3 \mathrm{H}), 1.45(\mathrm{~s}, 3 \mathrm{H}), 1.40(\mathrm{~s}, 3 \mathrm{H}), 1.26(\mathrm{br} \mathrm{s}$, $17 \mathrm{H}), 0.88(\mathrm{t}, J=6.8 \mathrm{~Hz}, 3 \mathrm{H}) ;{ }^{13} \mathrm{C}$ NMR $\left(100 \mathrm{MHz}, \mathrm{CDCl}_{3}\right) \delta$ $170.7,98.5,80.2,65.5,65.5,36.6,31.9,30.0,29.5,29.5,29.3$, $29.3,29.3,27.8,24.4,22.7,22.4,22.2,19.9,14.1$; HRMS (EI) $\mathrm{m} / \mathrm{z}$ calcd for $\mathrm{C}_{20} \mathrm{H}_{36} \mathrm{O}_{4}(\mathrm{M})^{+} 340.2614$, found 340.2607 .

\subsection{8. (4S)-hydroxytanikolide (2)}

$1 \mathrm{M} \mathrm{HCl}(3.2 \mathrm{~mL})$ was added dropwise to the solution of acetonide $(4 S)-34(219 \mathrm{mg}, 0.64 \mathrm{mmol})$ in THF $(3.2 \mathrm{~mL})$ at room temperature. After stirring the reaction mixture for $24 \mathrm{~h}$, the reaction mixture was quenched by saturated aqueous $\mathrm{NaHCO}_{3}(3$ $\mathrm{mL})$. The mixture was extracted with $\mathrm{Et}_{2} \mathrm{O}(3 \times 10 \mathrm{~mL})$ and combined organic layers were dried over anhydrous $\mathrm{Na}_{2} \mathrm{SO}_{4}$. The filtrate concentrated in vacuo, and the residue was purified by column chromatography (silica gel, 50\% EtOAc in hexane) to provide (4S)-hydroxytanikolide $(2,181 \mathrm{mg}, 94 \%)$ as a white solid: mp 79-80 ${ }^{\circ} \mathrm{C} ;[\alpha]_{\mathrm{D}}{ }^{20}+13.3\left(\mathrm{c} 1.0, \mathrm{CHCl}_{3}\right)$; IR $\left(\mathrm{KBr}, \mathrm{cm}^{-1}\right)$ v 3485, 3419, 2954, 2920, 2850, 1764, 1473, 1464, 1402, 1344, $1198,1112,1033,1011,990,955,821 ;{ }^{1} \mathrm{H}$ NMR $(400 \mathrm{MHz}$, $\left.\mathrm{CDCl}_{3}\right) \delta 4.53(\mathrm{t}, J=7.2 \mathrm{~Hz}, 1 \mathrm{H}), 3.78(\mathrm{dd}, J=11.6,4.0 \mathrm{~Hz}, 1 \mathrm{H})$ $3.61(\mathrm{dd}, J=11.6,9.0 \mathrm{~Hz}, 1 \mathrm{H}), 2.65-2.47(\mathrm{~m}, 3 \mathrm{H}), 2.37$ (ddd, $J=$ 18.0, 12.4, $9.2 \mathrm{~Hz}, 1 \mathrm{H}), 2.17(\mathrm{~m}, 1 \mathrm{H}), 2.08(\mathrm{dd}, J=8.8,4.0 \mathrm{~Hz}$, $1 \mathrm{H}), 1.51(\mathrm{~m}, 1 \mathrm{H}), 1.44-1.39(\mathrm{~m}, 2 \mathrm{H}), 1.26(\mathrm{br} \mathrm{s}, 17 \mathrm{H}), 0.88(\mathrm{t}, J$ $=6.7 \mathrm{~Hz}, 3 \mathrm{H}) ;{ }^{13} \mathrm{C} \mathrm{NMR}\left(100 \mathrm{MHz}, \mathrm{CDCl}_{3}\right) \delta 176.8,84.3,73.6$, $65.9,33.3,31.9,30.2,29.60,29.58,29.53,29.45,29.3,28.3,22.8$, 22.7, 21.9, 14.1; HRMS (EI) $\mathrm{m} / \mathrm{z}$ calcd for $\mathrm{C}_{17} \mathrm{H}_{32} \mathrm{O}_{4}(\mathrm{M})^{+}$ 300.2301 , found 300.2311 .
4.3.9. $(2 R, 3 R, 5 S)-5-($ tert-Butyldimethylsilyloxy)methyl-3methoxymethyloxy-2-(methoxymethyloxy)methyl-2allyltetrahydrofuran $\{(3 R)-35\}$

${ }^{i} \operatorname{Pr}_{2}$ NEt $(0.1 \mathrm{~mL}, 0.58 \mathrm{mmol})$ and $\mathrm{MOMCl}(0.03 \mathrm{~mL}, 0.41$ mmol) was added to the solution of diol (3R)-8a (35 mg, 0.12 mmol) in $\mathrm{CH}_{2} \mathrm{Cl}_{2}(0.6 \mathrm{~mL})$ at $0{ }^{\circ} \mathrm{C}$ under $\mathrm{N}_{2}$. After stirring at $0{ }^{\circ} \mathrm{C}$ for $1 \mathrm{~h}$, the reaction mixture was allowed to warm to room temperature. After stirring the reaction mixture for $3 \mathrm{~h},{ }^{i} \mathrm{Pr}_{2} \mathrm{NEt}$ $(0.01 \mathrm{~mL}, 0.06 \mathrm{mmol})$ and $\mathrm{MOMCl}(0.004 \mathrm{~mL}, 0.06 \mathrm{mmol})$ was added to the solution and the reaction mixture was stirred for $3 \mathrm{~h}$. The mixture was quenched by the addition of saturated aqueous $\mathrm{NaHCO}_{3}$ and the mixture was extracted with EtOAc $(3 \times 5 \mathrm{~mL})$. Combined organic layers were washed with water $(10 \mathrm{~mL})$ and brine $(10 \mathrm{~mL})$ and dried over anhydrous $\mathrm{Na}_{2} \mathrm{SO}_{4}$. The filtrate was concentrated in vacuo, and the residue was purified by column chromatography (silica gel, 10\% EtOAc in hexane) to provide (3R)-35 (38 mg, 84\%) as a colorless oil: $[\alpha]_{\mathrm{D}}{ }^{30}+6.3$ (c 0.87 , $\mathrm{CHCl}_{3}$ ); IR (neat, $\mathrm{cm}^{-1}$ ) v 3075, 2952, 2929, 2885, 2856, 2823, 2770, 1640, 1471, 1403, 1388, 1362, 1254, 1217, 1149, 1111, 1043, 919, 837, 777; ${ }^{1} \mathrm{H}$ NMR $\left(400 \mathrm{MHz}, \mathrm{CDCl}_{3}\right) \delta 5.94$ (dddd, $J$ $=17.2,9.6,8.4,6.4 \mathrm{~Hz}, 1 \mathrm{H}), 5.10(\mathrm{~d}, J=17.2 \mathrm{~Hz}, 1 \mathrm{H}), 5.07(\mathrm{~d}, J$ $=9.6 \mathrm{~Hz}, 1 \mathrm{H}), 4.68(\mathrm{~d}, J=9.6 \mathrm{~Hz}, 1 \mathrm{H}), 4.66(\mathrm{~d}, J=9.6 \mathrm{~Hz}, 1 \mathrm{H})$, $4.61(\mathrm{~d}, J=6.8 \mathrm{~Hz}, 1 \mathrm{H}), 4.59(\mathrm{~d}, J=6.8 \mathrm{~Hz}, 1 \mathrm{H}), 4.22(\mathrm{dd}, J=$ 6.4, $4.8 \mathrm{~Hz}, 1 \mathrm{H}), 4.18(\mathrm{~m}, 1 \mathrm{H}), 3.60$ (ddd, $J=14.4,10.6,4.0 \mathrm{~Hz}$, 2H), $3.46(\mathrm{~d}, J=10.0 \mathrm{~Hz}, 1 \mathrm{H}), 3.38(\mathrm{~s}, 3 \mathrm{H}), 3.35(\mathrm{~s}, 3 \mathrm{H}), 3.34$ (d, $J=10.0 \mathrm{~Hz}, 1 \mathrm{H}), 2.50(\mathrm{dd}, J=14.0,6.4 \mathrm{~Hz}, 1 \mathrm{H}), 2.36(\mathrm{dd}, J=$ 14.0, $8.4 \mathrm{~Hz}, 1 \mathrm{H}), 2.13(\mathrm{dt}, J=13.2,6.4 \mathrm{~Hz}, 1 \mathrm{H}), 2.02(\mathrm{ddd}, J=$ 13.2, 7.2, 4.8 Hz, 1H), 0.89 (s, 9H), 0.05 (s, 6H); ${ }^{13} \mathrm{C}$ NMR (100 $\left.\mathrm{MHz}, \mathrm{CDCl}_{3}\right) \delta 134.6,117.4,96.8,96.0,85.3,79.7,77.5,70.9$, $65.6,55.5,55.3,36.2,34.3,25.9,18.3,-5.3,-5.4$; HRMS (FAB) $\mathrm{m} / \mathrm{z}$ calcd for $\mathrm{C}_{19} \mathrm{H}_{39} \mathrm{O}_{6} \mathrm{Si}(\mathrm{M}+\mathrm{H})^{+} 391.2516$, found 391.2516.

\subsubsection{0. \\ (2R,3R,5S)-3-Methoxymethyloxy-2- (methoxymethyloxy)methyl-2-allyltetrahydrofuran-5-methanol $\{(3 R)-46\}$}

TBAF (1.0 $\mathrm{M}$ in THF, $1.4 \mathrm{~mL}, 1.42 \mathrm{mmol}$ ) was added to a solution of silyl ether (3R)-35 (503 mg, $1.29 \mathrm{mmol})$ in THF (13 $\mathrm{mL}$ ) at $0{ }^{\circ} \mathrm{C}$ under $\mathrm{N}_{2}$ and then the resulting mixture was stirred at room temperature. After stirring for $1.5 \mathrm{~h}$, the reaction mixture was diluted with EtOAc $(40 \mathrm{~mL})$ and washed with water $(15 \mathrm{~mL})$ and brine $(15 \mathrm{~mL})$, and dried over anhydrous $\mathrm{Na}_{2} \mathrm{SO}_{4}$. The filtrate was concentrated in vacuo, and the residue was purified by column chromatography (silica gel, 45\% EtOAc in hexane) to provide alcohol $(3 R)-46(354 \mathrm{mg}, 99 \%)$ as a colorless oil: $[\alpha]_{\mathrm{D}}{ }^{27}$ $+12.4\left(\right.$ c 1.0, $\left.\mathrm{CHCl}_{3}\right)$; IR (neat, $\left.\mathrm{cm}^{-1}\right) \vee 3469,2933,2889,2824$, 1639, 1217, 1150, 1110, 1041, 918; ${ }^{1} \mathrm{H}$ NMR (400 MHz, $\mathrm{CDCl}_{3}$ ) $\delta 5.93$ (dddd, $J=16.8,10.0,8.2,6.4 \mathrm{~Hz}, 1 \mathrm{H}), 5.11$ (d, $J=16.8$ $\mathrm{Hz}, 1 \mathrm{H}), 5.10(\mathrm{~d}, J=10.0 \mathrm{~Hz}, 1 \mathrm{H}), 4.69(\mathrm{~d}, J=6.4 \mathrm{~Hz}, 1 \mathrm{H}), 4.65$ (s, 2H), $4.64(\mathrm{~d}, J=6.4 \mathrm{~Hz}, 1 \mathrm{H}), 4.40$ (t, $J=6.8 \mathrm{~Hz}, 1 \mathrm{H}), 4.27$ (ddd, $J=10.9,5.2,2.8 \mathrm{~Hz}, 1 \mathrm{H}), 3.80(\mathrm{dt}, J=12.0,2.8 \mathrm{~Hz}, 1 \mathrm{H})$, $3.54(\mathrm{~d}, J=13.4 \mathrm{~Hz}, 1 \mathrm{H}), 3.52(\mathrm{~d}, J=13.4 \mathrm{~Hz}, 1 \mathrm{H}), 3.43(\mathrm{~m}, 1 \mathrm{H})$, $3.383(\mathrm{~s}, 3 \mathrm{H}), 3.378(\mathrm{~s}, 3 \mathrm{H}), 2.96(\mathrm{dd}, J=9.8,2.8 \mathrm{~Hz}, 1 \mathrm{H}), 2.45$ $(\mathrm{dd}, J=14.0,6.4 \mathrm{~Hz}, 1 \mathrm{H}), 2.32-2.23(\mathrm{~m}, 2 \mathrm{H}), 2.08$ (ddd, $J=12.9$, 8.0, 6.8 Hz, 1H); ${ }^{13} \mathrm{C}$ NMR $\left(100 \mathrm{MHz}, \mathrm{CDCl}_{3}\right) \delta$ 133.9, 118.0, 96.6, 96.4, 85.1, 78.6, 77.3, 70.5, 65.3, 55.6, 55.5, 36.9, 33.9; HRMS (FAB) $\mathrm{m} / \mathrm{z}$ calcd for $\mathrm{C}_{13} \mathrm{H}_{25} \mathrm{O}_{6}(\mathrm{M}+\mathrm{H})^{+}$277.1651, found 277.1652 .

4.3.11. (2R,3R,5S)-3-Methoxymethyloxy-2(methoxymethyloxy)methyl-2-allyltetrahydrofuran-5carboaldehyde $\{(3 R)-36\}$

A solution of DMSO $(85 \mathrm{mg}, 1.09 \mathrm{mmol})$ in $\mathrm{CH}_{2} \mathrm{Cl}_{2}(0.4 \mathrm{~mL})$ was dropped to a solution of oxalyl chloride (92 $\mathrm{mg}, 0.72 \mathrm{mmol}$ ) in $\mathrm{CH}_{2} \mathrm{Cl}_{2}(0.8 \mathrm{~mL})$ at $-78{ }^{\circ} \mathrm{C}$ under $\mathrm{N}_{2}$ over $15 \mathrm{~min}$. A solution of alcohol (3R)-46 (100 mg, $0.36 \mathrm{mmol})$ in $\mathrm{CH}_{2} \mathrm{Cl}_{2}(0.6 \mathrm{~mL})$ was then dropped to the reaction mixture over $15 \mathrm{~min}$. After stirring 
for $30 \mathrm{~min}$ at $-78{ }^{\circ} \mathrm{C}$, triethylamine $(0.25 \mathrm{~mL}, 1.81 \mathrm{mmol})$ was added to the reaction mixture and the mixture was allowed to warm to room temperature over $70 \mathrm{~min}$. After stirred at room temperature for $45 \mathrm{~min}$, the reaction mixture was quenched by the addition of saturated aqueous $\mathrm{NH}_{4} \mathrm{Cl}(10 \mathrm{~mL})$. The mixture was extracted with $\mathrm{CH}_{2} \mathrm{Cl}_{2}(3 \times 15 \mathrm{~mL})$ and combined organic layers were dried over anhydrous $\mathrm{Na}_{2} \mathrm{SO}_{4}$. The filtrate was concentrated in vacuo, and the residue was purified by column chromatography (silica gel, $45 \%$ EtOAc in hexane) to provide aldehyde $(3 R)-\mathbf{3 6}(73 \mathrm{mg}, 74 \%)$ as a colorless oil: $[\alpha]_{\mathrm{D}}{ }^{25}-5.1(c$ $1.0, \mathrm{CHCl}_{3}$ ); IR (neat, $\mathrm{cm}^{-1}$ ) $\vee 3445,2930,2890,2824,1732$, $1639,1217,1150,1110,1040,918 ;{ }^{1} \mathrm{H}$ NMR (400 MHz, $\mathrm{CDCl}_{3}$ ) $\delta 9.68(\mathrm{~d}, J=1.8 \mathrm{~Hz}, 1 \mathrm{H}), 5.91(\mathrm{ddd}, J=17.4,9.2,6.9 \mathrm{~Hz}, 1 \mathrm{H})$, $5.13(\mathrm{~d}, J=17.4 \mathrm{~Hz}, 1 \mathrm{H}), 5.11(\mathrm{~d}, J=9.2 \mathrm{~Hz}, 1 \mathrm{H}), 4.69(\mathrm{~d}, J=$ $6.9 \mathrm{~Hz}, 1 \mathrm{H}), 4.65(\mathrm{~d}, J=6.9 \mathrm{~Hz}, 1 \mathrm{H}), 4.61(\mathrm{~d}, J=6.9 \mathrm{~Hz}, 1 \mathrm{H})$, 4.59 (d, $J=6.9 \mathrm{~Hz}, 1 \mathrm{H}), 4.43(\mathrm{t}, J=7.5 \mathrm{~Hz}, 1 \mathrm{H}), 4.24$ (dd, $J=$ $5.6,4.8 \mathrm{~Hz}, 1 \mathrm{H}), 3.51(\mathrm{~d}, J=10.1 \mathrm{~Hz}, 1 \mathrm{H}), 3.44(\mathrm{~d}, J=10.1 \mathrm{~Hz}$, $1 \mathrm{H}), 3.39$ (s, 3H), $3.35(\mathrm{~s}, 3 \mathrm{H}), 2.54(\mathrm{dd}, J=14.2,6.4 \mathrm{~Hz}, 1 \mathrm{H})$, 2.39 (dd, $J=14.2,7.1 \mathrm{~Hz}, 1 \mathrm{H}), 2.40(\mathrm{~m}, 1 \mathrm{H}), 2.25$ (ddd, $J=13.2$, 8.0, 4.6 Hz, 1H); ${ }^{13} \mathrm{C}$ NMR (100 MHz, $\left.\mathrm{CDCl}_{3}\right) \delta 203.0,133.7$, 118.2, 96.7, 96.1, 87.2, 81.4, 78.7, 70.9, 55.8, 55.4, 36.7, 34.4; HRMS (EI) $\mathrm{m} / \mathrm{z}$ calcd for $\mathrm{C}_{13} \mathrm{H}_{22} \mathrm{O}_{6}(\mathrm{M})^{+} 274.1416$, found 274.1396.

4.3.12.

$(4 R, 5 S)-4-$ Methoxymethyloxy-5(methoxymethyloxy)methyl-5-allyltetrahydropyran-1-one $\{(4 R)$ 37)

Catalyst 29 (17 mg, $0.040 \mathrm{mmol}, 30 \mathrm{~mol} \%)$ and DBU (0.005 $\mathrm{mL}, 0.027 \mathrm{mmol}, 20 \mathrm{~mol} \%$ ) was added to the solution of aldehyde (3R)-36 $(37 \mathrm{mg}, 0.14 \mathrm{mmol})$ in toluene $(2.7 \mathrm{~mL})$ at room temperature under $\mathrm{N}_{2}$. After refluxing for $10 \mathrm{~h}$, the reaction mixture was quenched by the addition of saturated aqueous $\mathrm{NH}_{4} \mathrm{Cl}(3 \mathrm{~mL})$ at room temperature. The mixture was extracted with EtOAc $(3 \times 10 \mathrm{~mL})$ and combined organic layers were dried over anhydrous $\mathrm{Na}_{2} \mathrm{SO}_{4}$. The filtrate was concentrated in vacuo, and the residue was purified by column chromatography (silica gel, $40 \%$ EtOAc in hexane) to provide lactone $(4 R)-37(25 \mathrm{mg}$, $68 \%$ ) as a colorless oil: $[\alpha]_{\mathrm{D}}{ }^{24}+13.3\left(c 1.0, \mathrm{CHCl}_{3}\right)$; IR (neat, $\left.\mathrm{cm}^{-1}\right)$ v 2932, 2825, 1738, 1342, 1249, 1214, 1149, 1111, 1037, 918; ${ }^{1} \mathrm{H}$ NMR $\left(400 \mathrm{MHz}, \mathrm{CDCl}_{3}\right) \delta 5.90$ (dddd, $J=14.4,11.6$, 8.6, $6.4 \mathrm{~Hz}, 1 \mathrm{H}), 5.164(\mathrm{~d}, J=11.6 \mathrm{~Hz}, 1 \mathrm{H}), 5.161(\mathrm{~d}, J=14.4$ $\mathrm{Hz}, 1 \mathrm{H}), 4.76(\mathrm{~d}, J=7.6 \mathrm{~Hz}, 1 \mathrm{H}), 4.70(\mathrm{~d}, J=7.6 \mathrm{~Hz}, 1 \mathrm{H}), 4.61$ (s, 2H), $4.02(\mathrm{dd}, J=6.4,3.2 \mathrm{~Hz}, 1 \mathrm{H}), 3.60(\mathrm{~d}, J=10.6 \mathrm{~Hz}, 1 \mathrm{H})$, $3.53(\mathrm{~d}, J=10.6 \mathrm{~Hz}, 1 \mathrm{H}), 3.42(\mathrm{~s}, 3 \mathrm{H}), 3.35(\mathrm{~s}, 3 \mathrm{H}), 2.73-2.65$ (m, 2H), 2.57-2.49 (m, 2H), 2.21-2.04 (m, 2H); ${ }^{13} \mathrm{C}$ NMR (100 $\left.\mathrm{MHz}, \mathrm{CDCl}_{3}\right) \delta 170.2,131.7,119.4,96.8,96.0,85.2,70.9,69.7$, 56.1, 55.6, 37.9, 26.2, 22.1; HRMS (EI) m/z calcd for $\mathrm{C}_{13} \mathrm{H}_{22} \mathrm{O}_{6}$ $(\mathrm{M})^{+} 274.1416$, found 274.1440 .

4.3.13.

(4R,5S)-4-Methoxymethyloxy-5(methoxymethyloxy)methyl-5-(2-undecen-1-yl)tetrahydropyran-1one $\{(4 R)-47\}$

Grubbs 2 nd catalyst $(4.0 \mathrm{mg}, 0.005 \mathrm{mmol})$ was added to the solution of (4R)-37 (65 mg, $0.23 \mathrm{mmol})$ and 1-decene $(0.45 \mathrm{~mL}$, $2.37 \mathrm{mmol})$ in $\mathrm{CHCl}_{3}(3.3 \mathrm{~mL})$ at room temperature under $\mathrm{N}_{2}$. After refluxing for $4 \mathrm{~h}$, Grubbs 2 nd catalyst $(2.0 \mathrm{mg}, 0.002$ mmol) was added to the reaction mixture and the mixture was refluxed for $2 \mathrm{~h}$. Solvent was removed in vacuo, and the residue was purified by column chromatography (silica gel, 35\% EtOAc in hexane) to provide (4R)-47 (66 $\mathrm{mg}, 72 \%)$ as a colorless oil: $[\alpha]_{\mathrm{D}}{ }^{26}+13.5\left(\mathrm{c} 1.0, \mathrm{CHCl}_{3}\right)$; IR (neat, $\left.\mathrm{cm}^{-1}\right) \vee 2925,2854,1741$, $1457,1248,1212,1150,1111,1038,975,919 ;{ }^{1} \mathrm{H}$ NMR $(400$ $\left.\mathrm{MHz}, \mathrm{CDCl}_{3}\right) \delta 5.58-5.43(\mathrm{~m}, 2 \mathrm{H}), 4.75(\mathrm{~d}, J=6.8 \mathrm{~Hz}, 1 \mathrm{H}), 4.69$ $(\mathrm{d}, J=6.8 \mathrm{~Hz}, 1 \mathrm{H}), 4.60(\mathrm{~s}, 2 \mathrm{H}), 4.00(\mathrm{dd}, J=6.0,3.2 \mathrm{~Hz}, 1 \mathrm{H})$, $3.59(\mathrm{~d}, J=10.8 \mathrm{~Hz}, 1 \mathrm{H}), 3.52(\mathrm{~d}, J=10.8 \mathrm{~Hz}, 1 \mathrm{H}), 3.42(\mathrm{~s}, 3 \mathrm{H})$, 3.35 (s, 3H), 2.72-2.43 (m, 4H), 2.20-2.04 (m, 2H), 2.01 (dd, $J=$
13.0, $6.8 \mathrm{~Hz}, 2 \mathrm{H}), 1.26$ (br s, $12 \mathrm{H}), 0.88(\mathrm{t}, J=6.8 \mathrm{~Hz}, 3 \mathrm{H}) ;{ }^{13} \mathrm{C}$ NMR $\left(100 \mathrm{MHz}, \mathrm{CDCl}_{3}\right) \delta 170.4,135.7,134.0,122.6,121.9$, 96.8, 96.0, 85.7, 85.4, 71.0, 69.8, 56.1, 55.5, 36.6, 32.6, 31.9, $31.8,31.1,29.4,29.30,29.26,29.2,29.1,26.33,26.27,22.6,22.2$, 14.1; HRMS (EI) $\mathrm{m} / \mathrm{z}$ calcd for $\mathrm{C}_{21} \mathrm{H}_{38} \mathrm{O}_{6}(\mathrm{M})^{+} 386.2668$, found 386.2682 .

4.3.14. (4R,5S)-4-Methoxymethyloxy-5(methoxymethyloxy)methyl-5-undecanyltetrahydropyran-1-one $\{(4 R)-38\}$

$\mathrm{Pd} / \mathrm{C}\{2.2 \mathrm{mg}, 10 \mathrm{wt} \%$ of $(4 R)-47\}$ was added to the solution of $(4 R)-47(22 \mathrm{mg}, 0.057 \mathrm{mmol})$ in $\mathrm{MeOH}(0.3 \mathrm{~mL})$ at room temperature. The reaction mixture was vigorously stirred under $\mathrm{H}_{2}$ atmosphere for $10 \mathrm{~h}$. The mixture was filtered through a pad of Celite. The filtrate was concentrated in vacuo and the residue was purified by column chromatography (silica gel, 35\% EtOAc in hexane) to provide (4R)-38 (20 mg, 91\%) as a colorless oil: $[\alpha]_{\mathrm{D}}{ }^{26}-15.7\left(c 1.0, \mathrm{CHCl}_{3}\right)$; IR (neat, $\left.\mathrm{cm}^{-1}\right) \vee 2925,2854,1739$, 1466, 1252, 1214, 1150, 1113, 1036, 919; ${ }^{1} \mathrm{H}$ NMR (400 MHz, $\left.\mathrm{CDCl}_{3}\right) \delta 4.74(\mathrm{~d}, J=7.3 \mathrm{~Hz}, 1 \mathrm{H}), 4.69(\mathrm{~d}, J=7.3 \mathrm{~Hz}, 1 \mathrm{H}), 4.62$ $(\mathrm{s}, 2 \mathrm{H}), 4.03(\mathrm{dd}, J=6.9,3.2 \mathrm{~Hz}, 1 \mathrm{H}), 3.62(\mathrm{~d}, J=10.8 \mathrm{~Hz}, 1 \mathrm{H})$, $3.56(\mathrm{~d}, J=10.8 \mathrm{~Hz}, 1 \mathrm{H}), 3.41(\mathrm{~s}, 3 \mathrm{H}), 3.36(\mathrm{~s}, 3 \mathrm{H}), 2.67(\mathrm{dt}, J=$ $18.3,7.8 \mathrm{~Hz}, 1 \mathrm{H}), 2.52(\mathrm{dt}, J=18.3,6.4 \mathrm{~Hz}, 1 \mathrm{H}), 2.15(\mathrm{~m}, 1 \mathrm{H})$, $2.05(\mathrm{dt}, J=13.9,6.9 \mathrm{~Hz}, 1 \mathrm{H}), 1.85-1.72(\mathrm{~m}, 2 \mathrm{H}), 1.51-1.26(\mathrm{~m}$, $18 \mathrm{H}), 0.88(\mathrm{t}, J=7.3 \mathrm{~Hz}, 3 \mathrm{H}) ;{ }^{13} \mathrm{C}$ NMR $\left(100 \mathrm{MHz}, \mathrm{CDCl}_{3}\right) \delta$ $170.5,96.8,95.9,85.8,71.0,69.5,56.0,55.6,33.3,31.9,30.2$, $29.60,29.59,29.55,29.5,29.3,26.4,22.7,22.5,22.0$, 14.1; HRMS (FAB) $\mathrm{m} / \mathrm{z}$ calcd for $\mathrm{C}_{21} \mathrm{H}_{41} \mathrm{O}_{6}(\mathrm{M}+\mathrm{H})^{+}$389.2903, found 389.2921 .

\subsubsection{5. (4R)-hydroxytanikolide (2)}

$6 \mathrm{M} \mathrm{HCl}(0.23 \mathrm{~mL})$ was added dropwise to the solution of MOM ether (4R)-38 (54 mg, $0.14 \mathrm{mmol})$ in THF $(0.7 \mathrm{~mL})$ at room temperature. After stirring the reaction mixture at $50{ }^{\circ} \mathrm{C}$ for $3 \mathrm{~h}$, the reaction mixture was quenched by saturated aqueous $\mathrm{NaHCO}_{3}(2 \mathrm{~mL})$. The mixture was extracted with EtOAc $(3 \times 10$ $\mathrm{mL})$. Combined organic layers were washed with $\mathrm{H}_{2} \mathrm{O}(10 \mathrm{~mL})$ and brine $(10 \mathrm{~mL})$, and dried over anhydrous $\mathrm{Na}_{2} \mathrm{SO}_{4}$. The filtrate concentrated in vacuo, and the residue was purified by column chromatography (silica gel, 55\% EtOAc in hexane) to provide (4R)-hydroxytanikolide $(\mathbf{2}, 38 \mathrm{mg}, 90 \%)$ as a white solid: $\mathrm{mp} 50$ $52{ }^{\circ} \mathrm{C} ;[\alpha]_{\mathrm{D}}{ }^{26}-18.1\left(\mathrm{c} 1.0, \mathrm{CHCl}_{3}\right) ; \mathrm{IR}\left(\mathrm{KBr}, \mathrm{cm}^{-1}\right)$ v 3399, 3340, 2925, 2920, 2849, 1775, 1466, 1196, 1155, 1035; ${ }^{1} \mathrm{H}$ NMR (400 $\left.\mathrm{MHz}, \mathrm{CDCl}_{3}\right) \delta 4.55(\mathrm{t}, J=7.6 \mathrm{~Hz}, 1 \mathrm{H}), 3.62(\mathrm{~d}, J=11.2 \mathrm{~Hz}$, $1 \mathrm{H}), 3.56(\mathrm{~d}, J=11.2 \mathrm{~Hz}, 1 \mathrm{H}), 2.64-2.49(\mathrm{~m}, 2 \mathrm{H}), 2.33$ (ddd, $J=$ 18.2, 12.7, $9.2 \mathrm{~Hz}, 1 \mathrm{H}), 2.20(\mathrm{~m}, 1 \mathrm{H}), 2.15(\mathrm{~s}, 1 \mathrm{H}), 1.87(\mathrm{br} \mathrm{s}$, $1 \mathrm{H}), 1.68-1.58(\mathrm{~m}, 2 \mathrm{H}), 1.26($ br s, $18 \mathrm{H}), 0.88(\mathrm{t}, J=6.8 \mathrm{~Hz}$, $3 \mathrm{H}) ;{ }^{13} \mathrm{C}$ NMR $\left(100 \mathrm{MHz}, \mathrm{CDCl}_{3}\right) \delta 177.1,82.5,74.5,63.9,34.2$, $31.9,30.2,29.61,29.59,29.57,29.5,29.3,28.9,23.3,22.7,22.2$, 14.1; HRMS (EI) $\mathrm{m} / \mathrm{z}$ calcd for $\mathrm{C}_{17} \mathrm{H}_{32} \mathrm{O}_{4}(\mathrm{M})^{+} 300.2301$, found 300.2336 .

\subsection{Synthesis of nortanikolide (3)}

4.4.1. $(2 R, 5 S)$-2-Benzyloxymethyl-5-(tertbutyldimethylsilyloxy)methyl-2-undecanyltetrahydrofuran (39)

Potassium bis(trimethylsilyl)amide (KHMDS, $0.5 \mathrm{M}$ in toluene, $1.66 \mathrm{~mL}, 0.83 \mathrm{mmol}$ ) was added dropwise to the solution of alcohol 25 (166 mg, $0.41 \mathrm{mmol})$ in THF $(4.1 \mathrm{~mL})$ at $-20{ }^{\circ} \mathrm{C}$ under $\mathrm{N}_{2}$. After stirring for $30 \mathrm{~min}$ at $-20{ }^{\circ} \mathrm{C}$, benzyl bromide $(0.10 \mathrm{~mL}, 0.83 \mathrm{mmol})$ was added to the reaction mixture and the mixture was allowed to warm to $0{ }^{\circ} \mathrm{C}$. After stirred at $0{ }^{\circ} \mathrm{C}$ for $2 \mathrm{~h}$, the reaction mixture was quenched by the addition of saturated aqueous $\mathrm{Na}_{2} \mathrm{CO}_{3}(10 \mathrm{~mL})$. The mixture was extracted with EtOAc $(3 \times 20 \mathrm{~mL})$ and combined organic layers were dried over anhydrous $\mathrm{Na}_{2} \mathrm{SO}_{4}$. The filtrate was concentrated in vacuo, and the residue was purified by column chromatography (silica gel, 
3\% EtOAc in hexane) to provide benzyl ether 39 (178 $\mathrm{mg}, 88 \%)$ as a colorless oil: $[\alpha]_{\mathrm{D}}{ }^{26}-6.4\left(\mathrm{c} 1.0, \mathrm{CHCl}_{3}\right)$; IR (neat, $\left.\mathrm{cm}^{-1}\right) \mathrm{v}$ 2953, 2926, 2855, 1463, 1254, 1094, 836, 776; ${ }^{1} \mathrm{H}$ NMR (400 $\left.\mathrm{MHz}, \mathrm{CDCl}_{3}\right) \delta 7.33-7.28(\mathrm{~m}, 5 \mathrm{H}), 4.53(\mathrm{dd}, J=16.9,12.4 \mathrm{~Hz}$, 2H), 4.01 (ddd, $J=12.4,6.0,4.6 \mathrm{~Hz}, 1 \mathrm{H}), 3.64$ (dd, $J=10.5,4.1$ $\mathrm{Hz}, 1 \mathrm{H}), 3.51(\mathrm{dd}, J=10.5,6.0 \mathrm{~Hz}, 1 \mathrm{H}), 3.31(\mathrm{~s}, 2 \mathrm{H}), 1.98-1.87$ (m, 2H), 1.83-1.66 (m, 2H), $1.56(\mathrm{~m}, 2 \mathrm{H}), 1.25$ (br s, 18H) 0.88 (br s, 12H), $0.032(\mathrm{~s}, 3 \mathrm{H}), 0.027(\mathrm{~s}, 3 \mathrm{H}) ;{ }^{13} \mathrm{C}$ NMR $(100 \mathrm{MHz}$, $\left.\mathrm{CDCl}_{3}\right) \delta 138.7,128.2,127.5,127.4,85.1,79.9,75.1,73.4,65.9$, 37.3, 32.6, 31.9, 30.3, 29.68, 29.66, 29.6, 29.4, 29.3, 28.4, 25.9, 24.0, 22.7, 18.4, 14.1, -5.31, -5.33; HRMS (FAB) $\mathrm{m} / \mathrm{z}$ calcd for $\mathrm{C}_{30} \mathrm{H}_{55} \mathrm{O}_{3} \mathrm{Si}(\mathrm{M}+\mathrm{H})^{+}$491.3921, found 491.3885 .

\subsection{2. (2R,5S)-2-Benzyloxymethyl-2-undecanyltetrahydrofuran-5- methanol (40)}

TBAF (1.0 M in THF, $0.98 \mathrm{~mL}, 0.98 \mathrm{mmol})$ was added to a solution of silyl ether $39(456 \mathrm{mg}, 0.93 \mathrm{mmol})$ in THF $(9.3 \mathrm{~mL})$ at $0{ }^{\circ} \mathrm{C}$ under $\mathrm{N}_{2}$ and then the resulting mixture was stirred at room temperature. After stirring for $2 \mathrm{~h}$, the reaction mixture was diluted with EtOAc $(30 \mathrm{~mL})$ and washed with water $(15 \mathrm{~mL})$ and brine $(15 \mathrm{~mL})$, and dried over anhydrous $\mathrm{Na}_{2} \mathrm{SO}_{4}$. The filtrate was concentrated in vacuo, and the residue was purified by column chromatography (silica gel, 17\% EtOAc in hexane) to provide alcohol $40(337 \mathrm{mg}, 96 \%)$ as a colorless oil: $[\alpha]_{\mathrm{D}}{ }^{26}+4.2$ (c 1.0, $\mathrm{CHCl}_{3}$ ); IR (neat, $\mathrm{cm}^{-1}$ ) v 3452, 2925, 2853, 1464, 1363, $1206,1099,1077,1030,735,698 ;{ }^{1} \mathrm{H}$ NMR $\left(400 \mathrm{MHz}, \mathrm{CDCl}_{3}\right) \delta$ $7.33-7.28(\mathrm{~m}, 5 \mathrm{H}), 4.59(\mathrm{~d}, J=11.9 \mathrm{~Hz}, 1 \mathrm{H}), 4.52(\mathrm{~d}, J=11.9$ $\mathrm{Hz}, 1 \mathrm{H}), 4.16$ (ddd, $J=10.1,6.6,3.2 \mathrm{~Hz}, 1 \mathrm{H}), 3.79$ (dt, $J=11.4$, $3.2 \mathrm{~Hz}, 1 \mathrm{H}), 3.47$ (d, $J=9.2 \mathrm{~Hz}, 1 \mathrm{H}), 3.43$ (ddd, $J=11.4,8.9$, $3.2 \mathrm{~Hz}, 1 \mathrm{H}), 3.34(\mathrm{~d}, J=9.2 \mathrm{~Hz}, 1 \mathrm{H}), 2.92(\mathrm{dd}, J=8.9,3.2 \mathrm{~Hz}$, $1 \mathrm{H}), 2.10$ (ddd, $J=11.9,8.5,6.4 \mathrm{~Hz}, 1 \mathrm{H}), 2.04-1.90(\mathrm{~m}, 2 \mathrm{H})$, 1.72 (ddd, $J=11.9,8.2,6.9 \mathrm{~Hz}, 1 \mathrm{H}), 1.50-1.46(\mathrm{~m}, 2 \mathrm{H}), 1.25(\mathrm{br}$ $\mathrm{s}, 18 \mathrm{H}) 0.88(\mathrm{t}, J=6.9 \mathrm{~Hz}, 3 \mathrm{H}) ;{ }^{13} \mathrm{C}$ NMR $\left(100 \mathrm{MHz}, \mathrm{CDCl}_{3}\right) \delta$ $137.9,128.4,127.74,127.73,85.4,80.1,75.3,73.5,65.4,38.1$, $32.6,31.9,30.2,29.7,29.60,29.59,29.34,29.31,27.7,23.9,22.7$, 14.1; HRMS (FAB) $\mathrm{m} / \mathrm{z}$ calcd for $\mathrm{C}_{24} \mathrm{H}_{41} \mathrm{O}_{3}(\mathrm{M}+\mathrm{H})^{+} 377.3056$, found 377.3030 .

4.4.3. (S)-4-Benzyloxymethyl-4-undecanyltetrahydrofuran-1-one (42) and (S)-4-Benzoyloxymethyl-4-undecanyltetrahydrofuran-1one (43)

2-Iodobenzamide $\mathbf{4 1}^{27}(17.3 \mathrm{mg}, 0.06 \mathrm{mmol}$ ), additive (none or BHT, $13.2 \mathrm{mg}, 0.06 \mathrm{mmol})$ and Oxone ${ }^{\circledR}(612 \mathrm{mg}, 1.00 \mathrm{mmol})$ were added to a solution of tetrahydrofuran-2-methanol 40 (75 $\mathrm{mg}, 0.20 \mathrm{mmol})$ in DMF $(1.0 \mathrm{~mL})$ at room temperature. After completion of the reaction (checked by TLC), the mixture was diluted with EtOAc $(20 \mathrm{~mL})$ and quenched by water $(10 \mathrm{~mL})$. The organic layer was washed by saturated aqueous $\mathrm{Na}_{2} \mathrm{~S}_{2} \mathrm{O}_{3}(10$ $\mathrm{mL})$, saturated aqueous $\mathrm{NaHCO}_{3}(10 \mathrm{~mL})$, water $(10 \mathrm{~mL})$ and brine $(10 \mathrm{~mL})$, and dried over anhydrous $\mathrm{Na}_{2} \mathrm{SO}_{4}$. The filtrate was concentrated in vacuo, and the residue was purified by column chromatography (silica gel, $10 \%$ EtOAc in hexane) to provide lactone 42 (no additive: $47 \mathrm{mg}, 65 \%$; with BHT: $60 \mathrm{mg}$, $83 \%$ ) as a colorless oil and side product $\mathbf{4 3}$ (no additives: $9 \mathrm{mg}$, $12 \%$; with BHT: trace) as a colorless oil. 42 : $[\alpha]_{\mathrm{D}}^{22}+2.7(c 1.0$, EtOH); IR (neat, $\left.\mathrm{cm}^{-1}\right) \vee 2925,2854,1774,1455,1203,1162$, 1115, 942, 738, 698; ${ }^{1} \mathrm{H}$ NMR (400 MHz, $\left.\mathrm{CDCl}_{3}\right) \delta$ 7.37-7.27 (m, $5 \mathrm{H}), 4.58(\mathrm{~d}, J=11.9 \mathrm{~Hz}, 1 \mathrm{H}), 4.51(\mathrm{~d}, J=11.9 \mathrm{~Hz}, 1 \mathrm{H}), 3.53(\mathrm{~d}$, $J=10.1 \mathrm{~Hz}, 1 \mathrm{H}), 3.47(\mathrm{~d}, J=10.1 \mathrm{~Hz}, 1 \mathrm{H}), 2.72(\mathrm{ddd}, J=18.0$, 10.5, 8.7 Hz, 1H), 2.49 (ddd, $J=18.0,10.5,4.6 \mathrm{~Hz}, 1 \mathrm{H}$ ), 2.26 (ddd, $J=12.8,10.5,4.6 \mathrm{~Hz}, 1 \mathrm{H}$ ), 1.98 (ddd, $J=12.8,10.5,8.7$ $\mathrm{Hz}, 1 \mathrm{H}), 1.67-1.62(\mathrm{~m}, 2 \mathrm{H}), 1.25(\mathrm{br} \mathrm{s}, 18 \mathrm{H}) 0.88(\mathrm{t}, J=6.9 \mathrm{~Hz}$, $3 \mathrm{H}) ;{ }^{13} \mathrm{C}$ NMR $\left(100 \mathrm{MHz}, \mathrm{CDCl}_{3}\right) \delta 177.4,137.8,128.6,127.8$, 127.5, 87.7, 74.8, 73.6, 37.2, 31.9, 29.9, 29.64, 29.58, 29.5, 29.4, 29.32, 29.28, 28.8, 23.1, 22.7, 14.1, -0.02; HRMS (EI) $\mathrm{m} / \mathrm{z}$ calcd for $\mathrm{C}_{23} \mathrm{H}_{36} \mathrm{O}_{3}(\mathrm{M})^{+} 360.2665$, found 360.2645. 43: $[\alpha]_{D}{ }^{26}+6.1(c$
1.0, $\mathrm{CHCl}_{3}$ ); IR (neat, $\mathrm{cm}^{-1}$ ) v 2925, 2854, 1778, 1725, 1271, 1114, 943, 712; ${ }^{1} \mathrm{H}$ NMR (400 MHz, $\mathrm{CDCl}_{3}$ ) $\delta 8.00$ (dd, $J=6.9$, $1.4 \mathrm{~Hz}, 2 \mathrm{H}), 7.59(\mathrm{dd}, J=7.3,1.4 \mathrm{~Hz}, 1 \mathrm{H}), 7.46(\mathrm{dd}, J=7.3,6.9$ $\mathrm{Hz}, 2 \mathrm{H}), 4.45(\mathrm{~d}, J=11.9 \mathrm{~Hz}, 1 \mathrm{H}), 4.35$ (d, $J=11.9 \mathrm{~Hz}, 1 \mathrm{H})$, 2.73 (ddd, $J=18.3,10.5,8.2 \mathrm{~Hz}, 1 \mathrm{H}), 2.62(\mathrm{ddd}, J=18.3,10.5$, $5.0 \mathrm{~Hz}, 1 \mathrm{H}), 2.26$ (ddd, $J=13.3,10.5,5.0 \mathrm{~Hz}, 1 \mathrm{H}), 2.14$ (ddd, $J$ $=13.3,10.5,8.2 \mathrm{~Hz}, 1 \mathrm{H}), 1.82-1.78(\mathrm{~m}, 2 \mathrm{H}), 1.47-1.39(\mathrm{~m}, 2 \mathrm{H})$, $1.38-1.28(\mathrm{~m}, 5 \mathrm{H}), 1.26(\mathrm{br} \mathrm{s}, 11 \mathrm{H}) 0.88(\mathrm{t}, J=6.9 \mathrm{~Hz}, 3 \mathrm{H}) ;{ }^{13} \mathrm{C}$ NMR $\left(100 \mathrm{MHz}, \mathrm{CDCl}_{3}\right) \delta 176.5,166.1,133.4,129.6,128.6$, 86.3, 68.5, 37.2, 31.9, 29.8, 29.59, 29.57, 29.5, 29.4, 29.31, 29.27, $29.2,28.5,23.1,22.7,14.1$; HRMS (EI) $\mathrm{m} / \mathrm{z}$ calcd for $\mathrm{C}_{23} \mathrm{H}_{34} \mathrm{O}_{4}$ (M) 374.2457 , found 374.2454 .

\subsection{4. nortanikolide (3)}

$\mathrm{Pd}(\mathrm{OH})_{2} / \mathrm{C}(2.3 \mathrm{mg}, 10 \mathrm{wt} \%$ of $\mathbf{4 2})$ was added to the solution of $42(23 \mathrm{mg}, 0.06 \mathrm{mmol})$ in $\mathrm{MeOH}(0.8 \mathrm{~mL})$ at room temperature. The reaction mixture was vigorously stirred under $\mathrm{H}_{2}$ for $4 \mathrm{~h}$. The mixture was filtered through a pad of Celite. The filtrate was concentrated in vacuo and the residue was purified by column chromatography (silica gel, $45 \%$ EtOAc in hexane) to provide nortanikolide $(3,16 \mathrm{mg}, 94 \%)$ as a white solid: $\mathrm{mp} 43-$ $44{ }^{\circ} \mathrm{C} ;[\alpha]_{\mathrm{D}}{ }^{22}+9.8(c 1.0, \mathrm{EtOH})$; IR $\left(\mathrm{KBr}, \mathrm{cm}^{-1}\right)$ v 3427, 2955, 2917, 2848, 1749, 1469, 1297, 1218, 1078, 1048, 934; ${ }^{1} \mathrm{H}$ NMR $\left(400 \mathrm{MHz}, \mathrm{CDCl}_{3}\right) \delta 3.75(\mathrm{dd}, J=12.0,5.2 \mathrm{~Hz}, 1 \mathrm{H}), 3.54(\mathrm{dd}, J$ $=12.0,3.6 \mathrm{~Hz}, 1 \mathrm{H}), 2.70(\mathrm{ddd}, J=17.9,10.7,7.2 \mathrm{~Hz}, 1 \mathrm{H}), 2.58$ (ddd, $J=17.9,10.7,6.4 \mathrm{~Hz}, 1 \mathrm{H}), 2.04-1.96$ (m, 2H), 1.66 (br t, $J$ $=7.6 \mathrm{~Hz}, 1 \mathrm{H}), 1.30(\mathrm{br} \mathrm{s}, 5 \mathrm{H}), 1.25(\mathrm{br} \mathrm{s}, 15 \mathrm{H}) 0.88(\mathrm{t}, J=6.8 \mathrm{~Hz}$, $3 \mathrm{H}) ;{ }^{13} \mathrm{C} \mathrm{NMR}\left(100 \mathrm{MHz}, \mathrm{CDCl}_{3}\right) \delta 177.4,87.8,67.4,36.4,31.9$, 29.9, 29.6, 29.54, 29.50, 29.3, 27.4, 23.1, 22.7, 14.1; HRMS (EI) $\mathrm{m} / \mathrm{z}$ calcd for $\mathrm{C}_{16} \mathrm{H}_{30} \mathrm{O}_{3}(\mathrm{M})^{+} 270.2195$, found 270.2162 .

\section{Acknowledgments}

This research was supported, in part, by a Grant-in-Aid for Scientific Research (C) (Grant No. JP16K08158) from the Japan Society for the Promotion of Science (JSPS) and JSPS Core-toCore Program, B. Asia-Africa Science Platforms.

\section{Supplementary Material}

Supplementary data associated with this article can be found, in the online version, at http://...

\section{References and notes}

1. Singh IP, Milligan KE, Gerwick WH. J Nat Prod. 1999;62:1333-1335.

2. Cardllina JH. II, Moore RE, Arnold EV, Clardy J. J Org Chem. 1979;44:4039-4042.

3. (a) Kanada MR, Taniguchi T, Ogasawara K. Synlett. 2000:1019-1021; (b) Koumbis AE, Dieti KM, Vikentiou MG, Gallos JK. Tetrahedron Lett. 2003;44:2513-2516;

(c) Wu F, Hong R, Khan J, Liu X, Deng L. Angew Chem Int Ed. 2006;45:4301-4305.

4. Reddi RN, Prasad PK, Sundalai A. Org Lett. 2014;16:5674-5677.

5. (a) Sakito Y, Tanaka S, Asami M, Mukaiyama T. Chem Lett. 1980:12231226;

(b) Pougny JR, Rollin P, Sinaÿ P, Tetrahedron Lett. 1982;23:4929-4932; (c) Honda T, Imai M, Keiko K, Tsubuki M. J Chem Soc Perkin Trans 1. 1990;10:2677-2680;

(d) Carda M, Rodríguez S, Castillo E, Bellido A, Díaz-Oltra S, Marco JA. Tetrahedron. 2003;59:857-864;

(e) Zhang C, Hosoda N, Asami M. Tetrahedron Asymmetry. 2007; 18:2185-2189;

(f) Vichare P, Chattopadhyay A. Tetrahedron Asymmetry. 2008;19:598602 ;

6. (a) Ho PT, Wong S. Can J Chem. 1985;63:2221-2224;

(b) Giese B, Rupane R. Liebigs Ann Chem. 1987;3:231-233;

(c) Trinh MC, Florent JC, Monneret C. Tetrahedron. 1988;44:66336644; 
(d) Asaoka M, Hayashibe S, Sonoda S, Takei H. Tetrahedron. 1991;47:6967-6974;

(e) Trost BM, Tang W, Schulte JL. Org Lett. 2000;2:4013-4015;

(f) Mizutani H, Watanabe M, Honda T. Tetrahedron. 2002;58:89298936 ;

(g) Tanaka H, Kozuki Y, Ogasawara K. Tetrahedron Lett. 2002;43:4175-4178;

(h) Arasaki H, Iwata M, Makida M, Masaki Y. Chem Pharm Bull. 2004;52:848-852;

(i) Schomaker JM, Boran B. Org Biomol Chem. 2004;2:621-624;

(j) Ohgiya T, Nishiyama S. Tetrahedron Lett. 2004;45:8273-8275;

(k) Ohgiya T, Nakamura K, Nishiyama S. Bull Chem Soc Jpn. 2005;78:1549-1554;

(1) Kita Y, Matsuda S, Fujii E, Horai M, Hata K, Fujioka H. Angew Chem Int Ed. 2005;44:5857-5860;

(m) Fujioka H, Matsuda S, Horai M, Fujii E, Morishita M, Nishiguchi N, Hata K, Kita Y. Chem Eur J. 2007;13:5238-5248.

(n) Doran R, Duggan L, Singh S, Duffy CD, Guiry PJ. Eur J Org Chem. 2011:7097-7106;

(o) Doran R. Asymmetric Synthesis of Bioactive Lactones and the Development of a Catalytic Asymmetric Synthesis of $\alpha$-Aryl Ketones. Switzerland: Springer Theses; 2015:13-34.

7. (a) Gourder B, Lam WH. Angew Chem Int Ed. 2010;49:8733-8737

(b) Srivastava N, Reddy BVS. Helv Chim Acta. 2016;99:267-272.

8. (a) Enders D, Knopp M. Tetrahedron. 1996;52:5805-5818;

(b) Xie Y, Sun M, Zhou H, Cao Q, Gao K, Niu C, Yang H. J Org Chem. 2013;78:10251-10263.

9. Han X, Dong L, Geng C, Jiao P. Org Lett. 2015;17:3194-3197.

10. Miyamoto H, Iwamoto M, Nakada M. Heterocycles. 2005;66:61-68

11. Murai K, Nakamura A, Matsushita T, Shimura M, Fujioka H. Chem Eur J. 2012;18:8448-8453.

12. Akula R, Doran R, Guiry PJ. Eur J Org Chem. 2016;22:9938-9942.

13. (a) Maezaki N, Matsumori Y, Shogaki T, Soejima M, Tanaka T, Ohish H, Iwata C. Chem Commun. 1997:1755-1756;

(b) Maezaki N, Matsumori Y, Shogaki T, Soejima M, Ohishi H, Tanaka T, Iwata C. Tetrahedron. 1998;54:13087-13104;

(c) Wan Z, Nelson SG. J Am Chem Soc. 2000;122:10470-10471;

(d) Zhai H, Chen Q, Zhao J, Luo S, Jia X. Tetrahedron Lett. 2003;44:2893-2894;

(e) Chen Q, Deng H, Zhao J, Lu Y, Heb M, Zhai H. Tetrahedron. 2005;61:8390-8393;

(f) Yajima T, Saito C, Nagano H. Tetrahedron. 2005;61:10203-10215;

(g) Matsuo K, Hikita J, Nishiwaki K. Heterocycles. 2011;83:2601-2605;

(h) Zheng J, Lin L, Fu K, Zhang Y, Liu X, Feng X. Chem Eur J. 2014;20:14493-14498

14. (a) Pirrung MC, Werner JA. J Am Chem Soc. 1986;108:6060-6062;

(b) Roskamp EJ, Johnson CR. J Am Chem Soc. 1986;108:6062-6063;

(c) Murphy GK, West FG. Org Lett. 2006;8:4359-4361;

For reviews, see: (d) Padwa A, Weingarten MD. Chem Rev. 1996;96:223-269;

(e) Marmsäter FP, West FG. Chem Eur J. 2002;8:4346-4353;

(f) Murphy GK, Stewart C, West FG. Tetrahedron 2013;69:2667-2686;

(g) Sheng Z, Zhang Z, Chu C, Zhang Y, Wang J. Tetrahedron 2017;73:4011-4022.

15. For recent examples, see: (a) Jackson KL, Henderson JA, Motoyoshi H, Phillips AJ. Angew Chem Int Ed. 2009;48:2346-2350;

(b) Shimada N, Nakamura S, Anada M, Shiro M, Hashimoto S. Chem Lett. 2009;38:488-489;

(c) Clark JS, Berger R, Hayes ST, Thomas LH, Morrison AJ, Gobbi L. Angew Chem Int Ed. 2010; 49:9867-9870;

(d) Stewart C, McDonald R, West FG. Org Lett. 2011;13:720-723;

(e) Clark JS, Labre F, Thomas LH. Org Biomol Chem. 2011;9:4823-

4830;

(f) Hodgson DM, Man S. Chem Eur J. 2011;17:9731-9737;

(g) Clark JS, Vignard D, Parkin A. Org Lett. 2011;13:3980-3983;

(h) Clark JS, Berger R, Hayes ST, Senn HM, Farrugia LJ, Thomas LH, Morrison AJ, Gobbi L. J Org Chem. 2013;78:673-696;

(i) Clark JS, Yang G, Osnowski AP. Org Lett. 2013;15:1460-1463;

(j) Clark JS, Yang G, Osnowski AP. Org Lett. 2013;15:1464-1467;

(k) Hodgson DM, Moreno-Clavijo E, Day SE, Man S. Org Biomol Chem. 2013;11:5362-5369;

(1) Skrobo B, Deska J. Org Lett. 2013;15:5998-6001;

(m) Clark JS, Romiti F. Angew Chem Int Ed. 2013;52:10072-10075;

(n) Clark JS, Delion L, Farrugia LJ. Org Lett. 2014;16:4300-4303;

(o) Hodgson DM, Man S, Powell KJ, Perko Z, Zeng M, Moreno-Clavijo

E, Thompson AL, Moore MD. J Org Chem. 2014;79:9728-9734.

16. Yakura T, Muramatsu W, Uenishi J. Chem Pharm Bull. 2005;53:989-994.
17. Yakura T, Matsui K, Matsuzaka K, Yamashita M. Heterocycles. 2009;79:353-358.

18. Yakura T, Ozono A, Matsui K, Yamashita M, Fujiwara, T. Synlett. $2013 ; 24: 65-68$

19. For a preliminary communication on the total synthesis of $(+)$-tanikolide, see: Nambu H, Jinnouchi H, Fujiwara T, Yakura T. Synlett. 2016;27:1106-1109.

20. Hodgson and co-workers independently reported stereoselective dirhodium(II)-catalyzed oxonium ylide formation-rearrangement of 5allyloxy-2-diazo-3-oxocarboxylate. Hodgson DM, Man S, Powell KJ, Perko Z, Zeng M, Moreno-Clavijo E, Thompson AL, Moore MD. Org Lett. 2011;13:3980-3983.

21. Wang L, Thai K, Gravel M. Org Lett. 2009;11:891-893.

22. Yakura T, Horiuchi Y, Nishimura Y, Yamada A, Nambu H, Fujiwara T. Adv Synth Catal. 2016;358:869-873.

23. Szpilman AM, Cereghetti DM, Wurtz NR, Manthorpe JM, Carreira EM. Angew Chem Int Ed. 2008;47:4335-4338.

24. (a) Caglioti L, Magi M. Tetrahedron. 1963;19:1127-1131 (b) Hutchins RO, Milewski CA, Maryanoff BE. J Am Chem Soc. 1973;95:3662-3668

25. Ojha LR, Kudugunti S, Maddukuri PP, Kommareddy A, Gunna MR, Dokuparthi P, Gottam HB, Botha KK, Parapati DR, Vinod TK. Synlett. 2009:117-121

26. Krüger J, Hoffmann RW. J Am Chem Soc. 1997;119:7499-7504.

27. Yakura T, Yamada A, Noda N, Fujiwara T, Nambu H. Asian J Org Chem. $2014 ; 3: 421-424$ 